\title{
Abstracts of the 2015 Annual Meeting of the AANS/CNS Section on Disorders of the Spine and Peripheral Nerves
}

\author{
Phoenix, Arizona • March 4-7, 2015 \\ (DOI: 10.3171/2015.3.FOC-DSPNabstracts)
}

\begin{abstract}
100. Intraocular Pressure in Lumbar Spine Fusion Patients - A Randomized, Prospective Study

Sanford E. Emery, MD, MBA, Scott D.Daffner, MD, John C. France, $M D$ (West Virginia University, Department of Orthopaedics), Matthew Ellison, MD, Brian W. Grose, MD (West Virginia University, Department of Anesthesiology), Gerald R. Hobbs, PhD (West Virginia University, Department of Statistics), Nina B. Clovis (West Virginia University, Department of Orthopaedics)
\end{abstract}

Introduction: Ischemic optic neuropathy (ION) resulting in visual loss is a rare but devastating complication in spine surgery. Procedure time, blood loss, prone position and systemic factors may contribute to the risk of perioperative blindness. Elevated intraocular pressure (IOP) results in decreased perfusion and possibly ION. We evaluated the effect of head positioning on IOP in lumbar spine fusion patients.

Methods: Surgeries were performed on 52 patients at one institution. Inclusion criteria were lumbar spine fusion in patients 18-80 years old. Exclusion criteria included eye disease or injury, history of cervical stenosis, neck pain, trauma or current neoplasm. The control group had the head in neutral position with the face parallel to the level operating room table and the experimental group had the neck extended so the face had an angle of inclination of $10^{\circ}$ with the table. All patients were managed with Gardner-Wells tongs and ten pounds of traction on a Jackson table. Using an applanation tonometer, one author made all IOP measurements in pre-operative holding, supine after anesthetic induction, prone after positioning on the table and at regular intervals throughout the case. IOP measurements were recorded with respective time points and corresponding blood pressure and $\mathrm{CO} 2$ values. Independent variables included age, duration of procedure, blood loss, type/amount of fluid replacement, blood pressure, PCO2, gender and head position.

Results: Data were analyzed using ANOVA for categorical risk factors and with regression analysis for continuous risk factors. Mean values for IOP measurements in the prone position were statistically significantly lower in the $10^{\circ}$ elevated group versus the head-neutral group ( $\mathrm{p}=0.0014)$. No patient sustained visual loss or any cervical spine related complications.

Conclusion: Ten degree elevation of the head in the prone position for adult lumbar spine fusion patients resulted in statistically significantly lower IOP measurements compared to controls. As lower IOP correlates with increased optic nerve perfusion, this positioning intervention could mitigate the risk of perioperative blindness in spine surgery patients in the prone position.

101. Costs of Cervical Disc Replacement versus Anterior Cervical Discectomy and Fusion for Treatment of SingleLevel Cervical Disc Disease: An Analysis of the Blue Health Intelligence Database for Acute and Long-Term Costs and Complications

Kris Radcliff, MD (Rothman Institute and Thomas Jefferson University), Jeffrey Zigler, JD, Jack Zigler, MD (Texas Back Institute)

Introduction: The purpose of this study was to determine the reoperation rate, adverse event rate, as well as the direct and followon costs of cervical disc arthroplasty (CDA) compared to anterior cervical discectomy and fusion (ACDF) in a "real world" population of patients with single-level symptomatic cervical disc disease.

Methods: This was a retrospective, case-controlled study of a prospectively collected database of actual insurance industry allowed amounts (costs) and outcomes for patients age 18-60, who were continuously enrolled in one of 18 BlueCross BlueShield Association plans contributing data to the Blue Health Intelligence (BHI) claims database. Inclusion criteria were all patients who were treated surgically with either CDA or ACDF between January 2008 and December 2009 for single-level cervical pathology, who had pre-surgery claims reflecting at least six weeks of conservative care, without claims history of prior surgery.

Results: There were 6,962 patients who met inclusion criteria, including 6,635 ACDF patients and $327 \mathrm{CDA}$ patients. There were no statistically significant differences in the incidence of comorbidity between groups (ACDF $24.99 \%$ versus CDA $20.8 \%$, $p=0.0884$ Fisher's Exact test). Patient data was assessed for continuously enrolled patients, with post-operative claims data available as longterm as 48 months for approximately $23 \%$ of ACDF patients, and $24 \%$ of CDA patients. The mean follow-up was 25 months in both groups $(\mathrm{p}=0.7)$.

In the acute term, there was no statistically significant difference in pain events $(p=0.17)$ or readmission rates at 7,30 , or 90 days between groups $(\mathrm{p}>0.05)$. However, the mean total costs for the index operation and 90-day post-operative window were significantly reduced in the CDA patients ( $\$ 22,761$ versus $\$ 25,029, \mathrm{p}=0.0086$ )

By 24 months post-operative, there was a statistically significant increase in mechanical complications $(0.17 \%$ versus $0 \%)$ in the ACDF patients versus CDA patients. During the same follow-up period, there was a statistically significant increase in all costs for ACDF patients (CDA \$34,979.28 versus ACDF \$39,820.24, $\mathrm{p}=0.0008)$. By 36 months post-operative, the long-term relative rate of occurrence of cervical re-surgery events following ACDF surgery $(10.5 \%)$ was approximately twice that of the CDA group $(5.7 \%)$ (Hazard ratio, $\mathrm{p}=0.0214$ ).

Conclusion: Patients who undergo cervical disc arthroplasty for single-level degenerative disease have lower readmission rates, fewer mechanical complications, less index surgery and total future costs, and lower reoperation rates compared to those treated with ACDF. Excluding the cost of the index procedure, CDA is effective in reducing the monthly cost of care compared to ACDF, over an average of 2 years post-surgery. Patients with medical comorbidities in both groups had increased rates of readmission and increased costs compared to patients without comorbidities.

102. Laminoplasty vs Laminectomy and Fusion to Treat Cervical Spondylotic Myelopathy: Outcomes of the Prospective Multicenter AOSpine International CSM Study

Michael G. Fehlings MD PhD FRCSC FACS (Toronto Western Hospital), Carlo Santaguida MD FRCSC (University of Toronto), Ahmed G Ibrahim MBBS MRCS PhD (The National Hospital for Neurology and Neurosurgery), Lindsay Tetreault Bsc, Branko Kopjar, Shashank Sharad Kale MBBS MS MCh MD (All India Inst of Med Sciences), Helton L A Defino PhD (Faculdade de Medicina de Ribeirão Preto), Giuseppe Barbagallo MD (Policlinico University Hospital), Ronald HMA Bartels MD PhD (Radboud University Medical Center), Qiang Zhou MD (Southwestern Hospital), Paul M. Arnold MD (Department of Neurosurgery), Mehmet Zileli MD (1416 sok 7), Gamaliel Tan MD (Tan Tock Seng Hospital), 


\section{Meeting Abstracts: AANS/CNS Section on Disorders of the Spine and Peripheral Nerves}

Osmar Moraes MD, Yasutsugu Yukawa MD, Manuel Alvarado MD (Hospital San Juan de Dios), Massimo Scerrati MD (Via Conca 71), Tomoaki Toyone MD PhD (Teikyo University Chiba Medical Center), Masato Tanaka MD, Ciaran Bolger MD (Frenchay)

Introduction: Recent studies conducted in North America have demonstrated benefits of surgical treatment for symptomatic CSM. However, differences in pathology, comorbidities, treatment approaches and cultural response to treatment may affect the generalizability of these findings at the global level.

Methods: Patients receiving surgery for clinically symptomatic CSM were enrolled in a prospective multicenter, cohort study which is continuing to accrue subjects at 16 sites in Europe, Asia, North and South America. Subjects included were a part of a larger ongoing prospective observational study that has enrolled 492 subjects with CSM involving 16 clinical sites in Europe, Asia, North and South America. Of those, 108 received laminectomy and fusion; 66 received laminoplasty. The choice of surgical approach was at the discretion of the surgeon. Outcome measures were mJOA, the Nurick scale, NDI and the SF36 PCS and MCS Component Scores.

Results: Average age was 60.2 years (SD 10.8), 29.8\% were female. Subjects threated with laminectomy and fusion had more levels operated (5.0 vs. $4.4, \mathrm{P}<.01)$, shorter length of stay $(7.7 \mathrm{vs.}$ 15.7 days, $\mathrm{P}<.01)$ and, less severe neurologic impairment measured by mJOA $(12.6$ vs. $11.2, \mathrm{P}<.01)$. There were no differences in age, and baseline NDI, SF36v2 PCS and SF36v2 MCS. At 12 month follow-up, there were no differences in neurologic and functional outcomes for laminoplasty compared to laminectomy and fusion; mJOA (3.0 and 2.3, respectively, $\mathrm{P}=0.15$ ). Moreover, there were no differences in NDI (13.3 and 12.0, respectively, $\mathrm{P}=0.71)$, SF-36v2 PCS (8.5 and 7.7, respectively, $\mathrm{P}=0.66$ ) and SF-36v2 MCS (7.9 and 6.9 , respectively, $\mathrm{P}=0.56$ ).

Conclusions: Patients undergoing laminectomy and fusion and laminoplasty surgery for CSM show similar improvements in generic and disease specific outcome measures allowing for baseline differences in clinical presentation between the two groups of patients. Longer term follow-up will be required to determine whether any differences in outcome between the two forms of treatment emerge.

103. A Prospective, Multi-Center Assessment of the Best Versus Worst Clinical Outcomes for Adult Spinal Deformity (ASD) Surgery

Justin S. Smith MD PhD (University of Virginia Health System), Christopher I. Shaffrey MD FACS (University of Virginia), Virginie Lafage PhD (NYU Langone Medical Center), Frank Schwab $M D$, PhD, Themistocles Protopsaltis MD, Eric Klineberg MD (University of California - Davis), Munish Gupta MD (University of California - Davis; Ortho Surgery), Justin K Scheer BS (University of California, San Diego), Richard A. Hostin MD (Southwest Scoliosis Institute / Baylor Scoliosis Center), Kai-Ming G. Fu MD PhD (Weill Cornell Medical College), Alex Soroceanu, Robert Hart MD (Ohsu Spine Center), Douglas C. Burton MD, Shay Bess MD (Rocky Mountain Scoliosis and Spine Center), Christopher P. Ames $M D$ (University of California San Francisco Neuro Surgery)

Introduction: Average clinical outcomes are improved with surgery for selected ASD patients, but these outcomes span a broad range. Our objective was to compare ASD patients with best vs worst clinical outcomes to identify distinguishing factors.

Methods: Multicenter, prospective study of consecutive ASD patients treated operatively. Inclusion criteria included: age $>18 \mathrm{yr}$, ASD and min $2 \mathrm{yr}$ follow-up. Best vs worst outcomes patients were compared separately based on SRS-22 and ODI. Only those with BL SRS $-22<3.5$ or ODI $>30$ were included to minimize floor effect. Best and worst outcomes were defined for SRS-22 $(>4.5$ and $<2.5)$ and ODI $(<15$ and $>50)$.

Results: Of 227 patients, 187 had SRS-22<3.5 (25 best and 27 worst outcomes) and 162 had ODI $>30$ (43 best and 51 worst outcomes). Based on SRS-22, compared with best outcomes patients, those with worst outcomes had greater BL SRS-22 ( $\mathrm{p}<0.0001)$, higher prevalence of $\mathrm{BL}$ depression $(\mathrm{p}<0.001)$, greater comorbidities $(p=0.012)$, greater prevalence of prior surgery $(p=0.007)$, higher complication rate $(\mathrm{p}=0.012)$ and worse BL deformity (SVA $[\mathrm{p}=0.045]$, PI-LL mismatch $[\mathrm{p}=0.034])$. The best-fit multivariate model for SRS-22 included BL SRS-22 ( $\mathrm{p}=0.033)$, BL depression $(\mathrm{p}=0.012)$ and complications $(\mathrm{p}=0.030)$. Based on ODI, compared with best outcomes patients, those with worst outcomes had greater BL ODI $(\mathrm{p}<0.001)$, greater BL BMI $(\mathrm{p}=0.002)$, higher prevalence of BL depression $(p<0.028)$, greater BL SVA $(p=0.016)$, higher complication rate $(\mathrm{p}=0.02)$ and greater $2 y r$ SVA $(\mathrm{p}<0.001)$ and PI-LL mismatch $(\mathrm{p}=0.042)$. The best-fit multivariate model for ODI included BL ODI ( $<<0.001), 2 \mathrm{yr}$ SVA $(\mathrm{p}=0.014)$ and BL BMI $(p=0.037)$. Age did not distinguish best vs worst outcomes for SRS22 or ODI ( $>0.1)$.

Conclusions: Factors distinguishing best vs worst outcomes for ASD surgery included several patient factors (BL depression, BMI, comorbidities and disability), as well as residual deformity (2yr SVA) and complications. These findings suggest factors that may warrant further attention in order to achieve optimal surgical outcomes for ASD.

\section{Mayfield Basic Science Award} 104. Dual-gene Engineered Human Neural Stem Cells for the
Targeted Treatment of Experimental Spinal Cord Gliomas

Alexander E. Ropper MD (Barrow Neurological Institute), xiang zeng, Hariprakash Haragopal BTech (Brigham and Women's Hospital), Jamie Anderson, Dou Yu, Inbo Han MD Neurosurgeon, Hong J Lee, Seung U. Kim MD, PhD (San 5, Wonchon-dong), Yang D. Teng PhD MD

Introduction: There is no satisfactory treatment for malignant spinal cord gliomas (SCG). We used human neural stem cells (hNSCs), which possess the ability to track tumor cells, and engineered them to express enzymes for conversion of non-toxic, systemically administered pro-drugs into active local chemotherapeutic agents.

Methods: F3-hNSCs were designed to express two metabolic genes (F3.CD-TK: cytosine deaminase and thymidine kinase) that convert benign 5-fluorocytosine(5-FC) and ganciclovir (GCV) into oncolytic 5-FU and GCV-triphosphate. Immunodeficient RNU rats received injection of 10,000 G55 human glioblastoma cells at the C6 level, followed 7 days later by administration of F3.CD-TK or control cells (F3.CD or F3 debris) at $1 \mathrm{~mm}$ rostral and caudal to the tumor. 5-FC and GCV were administered by serial intraperitoneal injection. The primary endpoint was survival, and secondary endpoints included tumor growth and autonomic function.

Results: Rats $(\mathrm{n}=6)$ treated with F3.CD-TK plus 5-FC and GCV demonstrated increased survival (mean: $35.6 \pm 12.1$ days; $p$ less than $0.05)$ relative to controls receiving either F3.CD $(20.8 \pm 3.4$ days $)$ or cell debris $(19.8 \pm 4.0$ days). The speed of tumor growth was slower in the F3.CD-TK group $(0.573 \pm 0.11 \mathrm{~mm} 3 /$ day $)$ compared to cell debris controls $(0.941 \pm 0.02 \mathrm{~mm} 3 /$ day; p less than 0.05$)$. Pathological examination confirmed that F3-hNSCs migrated into the tumor mass and were in contact with glioma cells.

Conclusions: A novel approach of dual gene-altered hNSCs slowed the growth of SCG and increased survival, relative to control treatments. Our approach may offer a new therapy for spinal cord gliomas that can be used synergistically with other modalities. 


\section{Meeting Abstracts: AANS/CNS Section on Disorders of the Spine and Peripheral Nerves}

\section{Mayfield Clinical Science Award}

\begin{abstract}
105. A Cost-Effective Comparison Between Transforaminal and Lateral Lumbar Interbody Fusions Using the Incremental CostEffectiveness Ratio at 2 Year Follow-up.
\end{abstract}

Gurpreet Surinder Gandhoke MD (University of Pittsburgh Medical Center), HanMoe Shin (University of Pittsburgh), Yue Fang Chang PhD, Zachary J Tempel MD (UPMC), Peter C. Gerszten MD MPH FACS (Univiversity of Pittsburgh Physicians-Department of Neurosurgery), David O. Okonkwo MD PhD (University of Pittsburgh), Adam S. Kanter MD (University of Pittsburgh Medical Center Presbyterian)

Introduction: Both transforaminal lumbar interbody fusion (TLIF) and lateral lumbar interbody fusion (LLIF) are effective surgical interventions for appropriately selected patients with degenerative lumbar spondylosis. This study sought to compare health care costs associated with these procedures by calculating the incremental cost-effectiveness ratio (ICER) and, thereby, the difference in the mean total cost per quality adjusted life year (QALY) gained for TLIF versus LLIF for the treatment of degenerative spondylosis. We further calculated the thresholds for Minimum Clinically Important Difference and Minimum Cost Effective Difference for patient-reported outcome measures at 2-year follow-up.

Methods: Forty-five patients who underwent single level TLIF and 29 patients who underwent single level stand-alone LLIF for degenerative spondylosis with low back and leg pain were included. All costs from diagnosis through 2-year postsurgical follow up were available from a comprehensive single center data bank within a unified hospital system. Total cost to the third-party payor for all spine-related medical resource use from the time of diagnosis through 2 years was recorded. QALYs were calculated from EQ5D collected in an unbiased manner by a non-clinical staff member. Difference in mean total cost per QALY gained for LLIF minus that for TLIF was assessed as the incremental cost-effectiveness ratio (ICER: Cost LLIF- Cost TLIF/QALY LLIF- QALY TLIF).

Results: Significant improvements were observed at 2 year follow up for both TLIF and LLIF utilizing SF36PCS, ODI, VAS BP, VAS LP and EQ5D. ICER calculations revealed similar mean cumulative QALYs gained at the 2-year interval ( 0.67 for TLIF and 0.60 for LLIF; $p=0.331)$. Mean total cost of care following TLIF and LLIF were $\$ 53,038$ and $\$ 55,464$, respectively; $(p=0.960)$. MCED thresholds with an anchor of $<\$ 50,000 /$ QALY were higher than MCID thresholds (calculated using the Health Transition Index anchor) for all patient-reported outcome measures. Total mean cost and EQ5D were statistically equivalent between the 2 treatment groups.

Conclusions: Transforaminal lumbar interbody fusion (TLIF) and lateral lumbar interbody fusion (LLIF) produced equivalent 2 -year patient outcomes at an equivalent cost effectiveness profile.

\section{Cervical Disc Arthroplasty vs. Anterior Cervical Discectomy and Fusion: Seven-Year Outcomes from a Prospective, Multicenter Investigational Device Exemption Study}

Mark Edwin Shaffrey MD FAANS FACS (University of Virginia Health System), Matthew F Gornet MD (The Orthopedic Center of St. Louis), John Kenneth Burkus (Hughston Clinic, PC), Perry J. Argires MD, FACS

Introduction: This study compared the safety and efficacy of treatment with a low-profile, titanium ceramic composite ball-intrough cervical disc to treatment using control ACDF (plated fusion with allograft).

Methods: Prospectively-collected data from 20 investigational sites were compared to the 265 historical control ACDF patients in a prior cervical disc IDE study. The 280 investigational patients with single-level cervical disc disease with radiculopathy and/or myelopathy received a PRESTIGE LP® Disc.

Results: Patient follow up at 84 months was $75.0 \% / \mathrm{CDA}$, $68.7 \% / A C D F$. Statistically significant improvements vs. pre-op in NDI, neck/arm pain and SF-36 were achieved by 1.5 months in both groups and maintained up to 84 months. At 84 months, mean improvements in NDI were $40.1 \pm 23.3$ points for CDA and $31.9 \pm 21.8$ for ACDF patients. SF-36 PCS/MCS mean improvements at 84 months were $13.1 \pm 11.9 / 8.2 \pm 12.3$ points for CDA and $10.7 \pm 11.8 / 8.3 \pm 13.6$ points for ACDF. Neurological success was $92.8 \%$ for CDA and $79.7 \%$ for ACDF patients. Cumulatively up to 84 months, the percentages of CDA and ACDF patients who had an $\mathrm{AE}$ classified as possibly implant or implant/surgical procedure related were similar; 18 (6.4\%) CDA and $29(10.9 \%)$ ACDF patients had any second surgery at the index level. Following implantation of the CDA device, mean angular motion at the target level was maintained at $24\left(7.5^{\circ}\right)$ and $84\left(6.9^{\circ}\right)$ months.. At 84 months, $90.9 \%$ of CDA and $85.6 \%$ of ACDF patients were satisfied with the results of their treatment. Median return to work time for CDA patients was 40 days, vs. 60 for ACDF. At 84 months, $68.3 \%$ of CDA and $73.1 \%$ of ACDF patients were working.

Conclusions: This anterior low-profile CDA device maintains mean postoperative segmental motion while providing the potential for biomechanical stability. CDA patients reported significantly improved clinical outcomes vs. baseline, at least equivalent to standard-of-care ACDF, up to 84 months after surgery.

\section{Cost Analysis of Postoperative Cervical Sagittal Balance for Cervical Spondylotic Myelopathy}

Nikhil Nayak MD (University of Pennsylvania), Robert G. Whitmore MD (Lahey Hospital \& Medical Center), Marie Roguski MD (Tufts Medical Center), Edward C. Benzel MD (Cleveland Clinic Foundation), Jill Curran MS, Subu N. Magge MD (Lahey Clinic), Erica Fay Bisson MD FAANS (University of Utah Health Care), Ajit A. Krishnaney MD, FAANS (Cleveland Clinic Foundation), Michael P. Steinmetz MD (Center for Spine Health), William Butler MD (Massachusetts General Hospital), Robert F. Heary MD (Rutgers, New Jersey Medical School), Zoher Ghogawala MD FACS (Lahey Hospital and Medical Center)

Introduction: In patients with cervical spondylotic myelopathy (CSM), postoperative cervical sagittal balance (CSB), measured using the C2-7 sagittal vertical axis (SVA), is inversely correlated with improvements in health-related quality of life (HRQoL). We analyzed outcomes and up-front direct costs in these patients.

Methods: 64 CSM patients enrolled from five centers received ventral or dorsal decompression and fusion. An expert panel confirmed clinical equipoise for either procedure. Patient-reported outcomes and SVA measurements were recorded out to one year post-operatively. Direct costs were calculated from hospital charges using cost-to-charge ratios. Patients were dichotomized to favorable $($ SVA $<40 \mathrm{~mm})$ and unfavorable (SVA $>40 \mathrm{~mm}$ ) post-operative CSB cohorts.

Results: Patients with unfavorable CSB were more likely to be male $(\mathrm{p}=.01)$ and have had preoperative imbalance $(\mathrm{p}<.001)$. Both cohorts had an increase in average SVA post-operatively, although there was a significantly higher magnitude of worsening in the unfavorable cohort (7.7 vs. $3.5 \mathrm{~mm}, \mathrm{p}=.049)$. There was a trend towards a greater degree of improvement in SF-36 PCS scores in the favorable CSB cohort (8.8 vs. 3.6, $\mathrm{p}=.067)$. Average adjusted direct costs were higher in the favorable CSB cohort ( $\$ 27,789$ vs. $\$ 20,073, \mathrm{p}=.052$ ).

Conclusions: Maintaining or achieving favorable post-operative CSB demonstrates a trend towards greater improvements in HRQoL at the expense of higher initial hospital costs. Future studies with greater sample sizes, as well as a comprehensive cost analysis including outpatient expenses, are necessary to understand the economic impact of sagittal imbalance. 


\section{Meeting Abstracts: AANS/CNS Section on Disorders of the Spine and Peripheral Nerves}

\author{
108. Predictors of Readmission Within 30 Days After Spinal \\ Surgery: A Single-center, Multivariate Analysis
}

Ryan Khanna BS, Joseph L McDevitt, Jamal McClendon MD (Northwestern University Hospital), Zachary Adam Smith MD (Northwestern Dept. of Neurological Surgery), Nader S. Dahdaleh $M D$ (Northwestern University), Richard G. Fessler MD

Introduction: Unplanned readmissions within thirty days have been identified as significant sources of morbidity and cost, and readmission rates will likely impact publically available quality comparisons between providers in the future. Thus, spine surgeons must establish clinically relevant readmission rates and identify factors that are predictive of readmission risk in order to permit accurate comparisons, improve risk-stratification, and direct efforts to minimize readmissions.

Methods: Records from 1187 consecutive spinal surgeries at one institution were respectively reviewed to collect data that described the patient, surgery, hospital course, complications, and readmissions. Potential risk factors for readmission to the hospital within 30 days were first examined for association via bivariate analysis. Predictors found to be significant on bivariate analysis were further examined by a multivariate model. Readmission cases were independently reviewed by three attending spinal neurosurgeons using accepted definitions to determine if the readmissions were preventable.

Results: The overall readmission rate was $6.1 \%$, and of these readmissions, $43 \%$ were preventable. By bivariate analysis, factors that increased the risk of readmission included age, BMI, endocrine comorbidity, cardiac comorbidity, cancer, number of surgical levels, increased surgical time, admission to the ICU, and each additional hospital day. Undergoing a fusion operation, gender, and patients' insurance type were not significant predictors of readmission. Upon multivariate analysis, only three variables were found to be significant predictors of readmission: two or more patient comorbidities (OR: 3.72, 95\% CI: 1.62-8.56), an admission to the ICU (OR: 2.68, 95\% CI: 1.45-4.95), and each additional spinal level involved (OR: $1.11,95 \%$ CI: $1.02-1.21)$

Conclusions: Predictors for all-cause 30-day readmission following spinal procedures include number of spinal levels performed during the surgery, number of patient comorbidities, and admission to the ICU. Future work should develop best practices to modify medical risk factors and comorbidities to decrease 30-day readmission rates.

\section{Prognostic Factors for Survival in Surgical Series of Symptomatic Metastatic Epidural Spinal Cord Compression: A Prospective North American Multi-Centre Study in 145 patients}

Anick Nater-Goulet MD (University of Toronto Western Hospital), Michael G. Fehlings MD PhD FRCSC FACS (Toronto Western Hospital), Lindsay Tetreault Bsc, Branko Kopjar, Paul M. Arnold MD (Department of Neurosurgery), Mark B. Dekutoski MD (The Mayo Clinic), Joel Finkelstein MD (Sunnybrook Health Sciences Center), Charles Fisher MD (Vancouver Spine Surgery Institute), John France MD, Laurence D. Rhines MD (University of Texas-MD Anderson Cancer Center), James M. Schuster MD (University of Pennsylvania), Alexander R. Vaccaro MD, Peter Rose

Introduction: Symptomatic Metastatic Epidural Spinal Cord Compression (MESCC) afflicts up to $10 \%$ of cancer patients. MESCC is associated with shortened survival and worsened quality of life. In a multidisciplinary setting, life expectancy and other factors, such as patient's symptoms and overall medical condition, are evaluated to select the optimal patient-specific treatment plan. This study aims to identify the key survival prognostic factors in surgically treated symptomatic MESCC patients.

Methods: 145 MESCC patients were enrolled in a prospective
North American multi-center study and followed postoperatively for 12 months. Using univariate analyses, Kaplan-Meier methods, and log-rank tests the prognostic value of various clinical predictors were assessed. Predictors with $\mathrm{p}<0.2$ in univariate analyses were included in the final Cox proportional hazards model.

Results: The overall median survival was 7.7 months; lung and breast cancer had the shortest and longest median survival, respectively (4.5 vs. 12.1 months). Nine patients $(6 \%)$, whose primary cancer were lung (3), kidney (3), melanoma (1), prostate (1), and breast (1), died within 30-days postoperatively; 46 (32\%), $61(42 \%)$, $74(51 \%)$, and $86(59 \%)$ died at 3, 6, 9, and 12 months, respectively. Significant predictors on the univariate analyses were: site of primary tumor, number of vertebrae involved, metastases outside the spine, bladder dysfunction, ability to walk 4 steps, ODI, EQ-5D, and ASIA scores; only spinal metastasis involving ? 4 vertebral bodies was included in the final model ( $p=0.002$, HR: 25).

Conclusions: The extent of spinal metastasis, which is regarded as an indicator of the severity of patient's metastasis burden, is an independent predictor of poor prognosis in patients with a single level symptomatic MESCC lesion. MESCC patients are a challenging heterogenous population. It is essential to identify factors predicting survival, functional, and clinical outcomes in this population to guide patient-centred management to optimize quality of life.

\section{Predicting Occurrence of Spine Surgery Complications Using 'Big Data' Modeling of an Administrative Claims Database}

John K. Ratliff MD FACS (Stanford University Medical Center), Lu Tian PhD, Anand Veeravagu MD (Stanford University School of Medicine), Tyler Scott Cole (Stanford Medical School), Richard Olshen PhD

Introduction: Post-operative metrics are increasingly important in determining standards of quality for physicians and hospitals. Little is known about what contributes to variation in these health outcomes in spinal surgery. Incorporating procedural and patient variables into a predictive model of adverse event occurrence in spine surgery would significantly advance the field.

Methods: We used longitudinal prospective data from a claims database and developed a prediction rule incorporating occurrence and type of complications. We structured our modeling to assess preoperative diagnosis, patient comorbidities, location in the spine, anterior versus posterior approach, fusion status, instrumentation status, number of levels, and use of BMP. We assessed a variety of adverse events. Prediction models were built using logistic regression with additive main effects and logistic regression with main effects as well as all two and three factor interactions. LASSO regularization was used to select features. Validation approaches included boosted additive trees and the CART algorithm. All tuning parameters were selected via cross-validation. Final prediction was evaluated by estimated AUC (area under a receiver operating characteristic curve) as predictions were applied to validation data.

Results: The model was developed from 279,145 records with minimum follow-up of 30 days. Preliminary assessment showed an adverse event rate of $13.95 \%$, well within norms found in the literature. We used the first 179,145 records for training (to predict adverse events) and the remaining 100,000 records for validation. There was remarkable similarity among methods, with AUC being about 0.70 for predicting the occurrence of adverse events (Figure 1). Correlations between predictive scores derived from the various methods were high, averaging 0.96 .

Conclusions: We present a modelling effort that predicts the occurrence and type complications in spine surgery. This effort reliably predicts complication occurrence based on administrative data. 


\section{Meeting Abstracts: AANS/CNS Section on Disorders of the Spine and Peripheral Nerves}

\section{How to Predict Return to Work After Lumbar Discectomy: Answers from the NeuroPoint-SD Registry}

Khoi Duc Than MD (UCSF Department of Neurological Surgery), Jill Curran MS, Daniel K. Resnick MD (University of WisconsinSchool of Medicine), Christopher I. Shaffrey MD FACS (University of Virginia), Zoher Ghogawala MD FACS (Lahey Hospital and Medical Center), Praveen V. Mummaneni MD (University of California San Francisco Spine Center)

Introduction: To date, the factors that predict whether a patient returns to work after lumbar discectomy are unknown. Information on postoperative work status is important in analyzing a procedure's cost effectiveness.

Methods: An observational prospective cohort study was completed at 13 academic and community sites (NeuroPoint-SD registry). Patients undergoing single-level lumbar discectomy were included. Variables assessed included age, gender, BMI, SF-36 physical function score, ODI, diabetes, smoking status, systemic illness, workers compensation status, and preoperative work status. The primary outcome was working status within three months after surgery. Logistic regression analysis was performed to determine which factors were predictive of return to work at three months following discectomy.

Results: There were 127 patients (out of 148 total) with data collected at three months postoperatively. Average age was 46 +/- 1 years. $66.9 \%$ of patients were working at three months postoperatively. Statistical analysis demonstrated that those more likely to return to work were: patients of younger age (44.5 years vs. 50.5 years, $\mathrm{p}=0.008)$, males $(55.3 \%$ vs. $28.6 \%, \mathrm{p}=0.05)$, those with higher SF-36 physical function scores $(44.0$ vs. $30.3, \mathrm{p}=0.02)$, those with lower ODI (43.8 vs. 52.6, $\mathrm{p}=0.01)$, non-smokers $(83.5 \%$ vs. $66.7 \%, \mathrm{p}=0.03)$, and those who were working preoperatively ( $91.8 \%$ vs. $26.2 \%, \mathrm{p}<0.0001)$. When controlling for those who were working preoperatively (105 patients), only age was a statistically significant (but likely not clinically significant) predictor of postoperative return to work (44.1 years vs. 51.1 years, $\mathrm{p}=0.049$ ).

Conclusions: In this cohort of lumbar discectomy patients, preoperative working status was the strongest predictor of postoperative working status three months after surgery. Factors not influencing return to work in the logistic regression analysis included gender, BMI, SF-36 physical function score, ODI, diabetes, smoking status, systemic illness, and workers compensation status.

112. Comparative Clinical and Economic Outcomes of Minimally Invasive Surgery for Posterior Lumbar Fusion: A Systematic Review and Meta-Analysis

Christina Goldstein MD, FRCSC, Kevin Macwan BHSc, Kala Sundararajan BSc, MSc, Raja Rampersaud MD, FRCSC (Toronto Western Hospital, University of Toronto)

Introduction: The comparative effectiveness and economic benefit of minimally invasive surgery (MIS) for lumbar fusion remains unclear.

Objective: The main objective of this systematic review and meta-analysis is to summarize the results of comparative effectiveness and economic research comparing MIS transforaminal lumbar interbody fusion (TLIF) to traditional open midline techniques.

Methods: A systematic review of Medline, EMBASE, Pubmed, Web of Science, and Cochrane databases was performed. A hand search of reference lists was performed. Studies were reviewed by 2 independent assessors to identify randomized controlled trials or comparative cohort studies including $\geq 10$ patients undergoing open or MIS TLIF for degenerative pathology and reporting at least one of: clinical outcome measure; perioperative outcome measure; radiographic outcome; complications; or economic analysis. Study quality was assessed using the GRADE protocol. A meta-analysis was conducted on outcomes data when appropriate.

Results: The systematic review and reference list search identified 3,306 articles, of which 26 met our inclusion criteria. All studies were of low or very low quality. The cohorts ( $n=856$ MIS / $\mathrm{n}=806$ open) were comparable in age, gender, surgical levels and diagnosis. Meta-analysis revealed changes in the perioperative outcomes of mean estimated blood loss, time to ambulation and length of stay favoring an MIS approach by $260 \mathrm{ml}(\mathrm{p}<0.00001), 3.5$ days $(p=0.0006)$ and 2.9 days $(p<0.00001)$ respectively. Operative time was not significantly different between the surgical techniques $(p=0.78)$. There was no significant difference in surgical adverse events $(\mathrm{p}=0.97)$, however, MIS cases were significantly less likely to experience medial adverse events $(\mathrm{RR}[95 \% \mathrm{CI}] \mathrm{MIS}$ vs. open = 0.39 [0.23-0.69], $\mathrm{p}=0.001)$. No difference in non-union $(\mathrm{p}=0.97)$ or reoperation rates $(p=0.97)$ was observed. At a median follow-up time of 24 months mean Oswestry Disability Index scores were slightly better in the MIS patients (mean difference MIS vs open = $3.32, \mathrm{p}=0.001$ ). Limited economic comparisons (3 studies) consistently showed decreased direct hospital costs associated with an MIS technique (range $=10.6-23.9 \%$ ).

Conclusions: Pooled analysis of the current literature comparing MIS to open TLIF for degenerative lumbar pathology suggests improved clinical and perioperative outcomes associated with decreased direct health care costs. Thus, there appears to be value associated with the performance of MIS lumbar fusion in this patient population.

113. Long-term clinical and occupational outcomes after long segmental arthrodesis for spinal deformity: The Effect of BMI and Smoking

Dennis Crandall, Jan Revella, Ken Schmidt (Sonoran Spine Center), R. McLemore (Mayo Clinic)

Introduction: Multilevel arthrodesis involved in correcting spinal deformity is associated with more complications than short segment spinal fusions. The pre-operative risk factors for complications and inferior outcomes in these patients are not clearly understood. We studied the impact of 2 specific risk factors, smoking and body mass, on the long-term clinical and occupational outcomes after arthrodesis for spinal deformity.

Methods: Prospectively collected data were retrospectively reviewed from 251 patients, age 63(18-83years) who underwent open posterior instrumented fusion with BMP for spinal deformity from 2002-2011 at one spine center. All had minimum 2 year clinical and radiographic follow-up. Fusions averaged 8 levels; 153 had TLIF, 61 had ALIF. The BMI and smoking status of the patient were recorded. Clinical outcomes included peri-operative and long-term complications, preoperative and 2 year Visual Analog Pain Scale (VAS) and Oswestry Disability Index (ODI). Return to work and the ability to remain working long-term were also analyzed, along with job lifting requirements: light, medium, or heavy. Using logistic regression, the association of BMI and smoking status on outcome measures was analyzed.

Results: At an average follow-up of 5 years(24-131months). Complications included nonunion-20, infection-16, adjacent fractures-28, long-term adjacent degeneration-73. Revision or extension of fusion was required in $68(27 \%)$. The average BMI was 26.1 (15.5-45.1) with 29 patients who were smokers. The average preoperative VAS and ODI were $6(0-10)$ and $48.16 \%(0.00-94.0)$, respectively. At 2 years post-operative the average VAS and ODI were $3.1(0-10)$ and $27.8 \%(0.0-84.0)$ respectively. Logistic regression found that neither BMI nor smoking were significant predictors of post-operative VAS and ODI scores. The only significant predictor of post-operative VAS and ODI scores were preoperative VAS and ODI scores. Time to return to work and the ability to remain at work long-term was similar smokers vs. nonsmokers. Though not 


\section{Meeting Abstracts: AANS/CNS Section on Disorders of the Spine and Peripheral Nerves}

statistically significant, obese patients took longer to return to work. The overall long-term complication rate was $30 \%$ in this study. Smoking and BMI had an Odds Ratio of 1.26 (95\% CI 0.54-2.9) and 1.05 (1.00-1.10) for rate of complications.

Conclusions: At an average 5 years follow-up after multi-segmental arthrodesis for spinal deformity, one quarter of the cohort had undergone additional surgery for adjacent level stenosis, fracture, or instability. While both smoking and obesity were associated with an increased risk of complications, the difference was not statistically significant.

200. Operative versus Non-operative Management In Elderly Patients over 75 years Undergoing Reconstructive Spinal Deformity Surgery

Daniel M. Sciubba BS MD (Johns Hopkins University), Justin K Scheer BS (University of California, San Diego), Justin S. Smith MD PhD (University of Virginia Health System), Eric Klineberg MD (University of California - Davis), Munish Gupta MD (University of California - Davis; Ortho Surgery), Gregory Mundis MD (San Diego Center for Spinal Disorders), Themistocles Protopsaltis MD, Virginie Lafage PhD (NYU Langone Medical Center), Han Jo Kim MD, Tyler R. Koski MD (Northwestern University), Khaled Kebaish $M D$, Christopher I. Shaffrey MD FACS (University of Virginia), Shay Bess MD (Rocky Mountain Scoliosis and Spine Center), Robert Hart MD (Ohsu Spine Center), Frank Schwab MD, PhD, Christopher P. Ames MD (University of California San Francisco Neuro Surgery)

Introduction: Elderly adult spinal deformity (ASD) patients often report worse health-related quality of life (HRQOL) than younger patients. Increasing age is associated with increased surgical morbidity. The objective of the present study was to compare the outcomes of patients with ASD over 75 years of age managed operatively (OP) and non-operatively (NONOP).

Methods: A retrospective review of a multicenter prospective ASD database was conducted. Inclusion criteria included age $=75 y r s$, ASD . Demographics, co-morbidities, radiographs, complications and HRQOL measures collected included pain with visual analog scale (VAS), Oswestry Disability Index (ODI), Short Form36(SF-36), and Scoliosis Research Society-22 (SRS22) at baseline, 1 and 2 yrs.

Results: 32 patients met criteria (OP:17, NONOP:15), all had a minimum $2 \mathrm{yr}$ clinical and radiographic follow-up. 13 out of the 32 were at least 80 years of age. There were no significant differences between OP and NONOP for age, BMI, and Charlson Comorbidity Index ( $>0.05$ for all). OP had worse baseline ODI, PCS, SRS Activity, Appearance, and Total than NONOP ( $p<0.05$ for all). There were no significant differences in any baseline radiographic parameters. OP had significant improvement in LL, C7SVA, PI-LL, ODI, PCS, and all SRS subdomains except mental compared with preop ( $\mathrm{p}<0.05$ for all). OP had significantly higher $2 \mathrm{yr}$ SRS appearance and Satisfaction $(\mathrm{p}<0.05)$ than NONOP. There were no significant differences between baseline and $2 \mathrm{yr}$ HRQOL for NONOP ( $>0.05) .10 / 17(58.8 \%)$ OP patients had at least 1 complication with a total of 27 complications including a $64.7 \%$ rate of PJK and a $35.3 \%$ rate of reoperation.

Conclusions: Elderly patients $=75$ years undergoing ASD surgery had significantly improved HRQOL and radiographic measures over baseline at two years compared to a similar non-operative cohort, despite substantial operative morbidity. This data may be used for preoperative patient counseling in this high-risk elderly population.
201. The Influence of Spinopelvic Alignment on Development of Symptomatic Adjacent Level Disease Following Single Level Lumbar Fusion

Zachary J Tempel MD (UPMC), Bryan D Bolinger DO (University of Pittsburgh Medical Center), Gurpreet Surinder Gandhoke (University of Pittsburgh Medical Centre), Phillip Parry MD (University of Pittsburgh Medical College), David O. Okonkwo MD PhD (University of Pittsburgh), Adam S. Kanter MD (University of Pittsburgh Medical Center Presbyterian)

Introduction: The annual incidence of developing symptomatic adjacent level disease (ALD) following lumbar fusion surgery ranges from 0.6 to $3.9 \%$. It has been suggested that sagittal malalignment may contribute to the development of ALD. We describe the relationship between spinopelvic parameters and symptomatic ALD requiring revision surgery following single-level transforaminal lumbar interbody fusion (TLIF).

Methods: All patients who underwent a single-level TLIF at either L4/5 or L5/S1 at our institution for between July 2006 and December 2012 were analyzed for pelvic tilt (PT), sacral slope (SS), pelvic incidence (PI), lumbar lordosis (LL), and PI-LL mismatch. Using univariate and multivariate logistic regression analysis, we compared the spinopelvic parameters of patients who required revision surgery for symptomatic ALD against those who did not. The sensitivities and specificities for symptomatic ALD requiring surgery were based on preoperative values of PT $>24 \mathrm{o}$ and pelvic mismatch $>12 \mathrm{o}$.

Results: 159 patients met inclusion criteria. The results of multivariate logistic regression analysis noted preoperative PI-LL mismatch (OR: 1.25 CI: 95\% (1.16, 1.33) p < 0.0001), postoperative PI-LL mismatch (OR: 1.40 CR: 95\% $(1.27,1.54) \mathrm{p}<0.0001)$, and postoperative change in LL (OR: $0.96, \mathrm{CI}: 95 \%(0.92,1.0) \mathrm{p}=0.39)$ to be statistically-significant independent predictors of symptomatic ALD requiring revision surgery. The sensitivity and specificity of a PI-LL mismatch $>120$ for symptomatic ALD requiring surgery following a single-level TLIF were $81.82 \%$ and $86.54 \%$. The positive predictive value for a postoperative PI-LL mismatch $=12 \mathrm{o}$ for symptomatic ALD was $79.0 \%$; the area under the receiver operating characteristic (ROC) curve was 0.92 .

Conclusions: Abnormal preoperative spinopelvic parameters and persistent mismatch are associated with higher rates of symptomatic ALD requiring surgery following a single-level TLIF. Postoperative PI-LL mismatch was the strongest predictor. Accounting for spinopelvic parameters in the preoperative period may improve outcome following single-level TLIF.

\section{Cost and Quality of Life Implications of Adjacent Segment Disease Following Lumbar Fusion}

Vincent J Alentado BS (Case Western Reserve University School of Medicine), Daniel Lubelski, Andrew T Healy MD, Jacob A. Miller BS (Cleveland Clinic Lerner College of Medicine), Douglas Orr, Michael P. Steinmetz, MD (Center for Spine Health), Edward C. Benzel MD (Cleveland Clinic Foundation), Thomas E. Mroz MD (Cleveland Clinic - Neuroscience Institute)

Introduction: Revision surgery for adjacent segment disease (ASD) has been shown to improve quality of life (QOL) a costeffective manner. However, the QOL and financial implications of developing ASD have not been well described in the literature.

Methods: Individuals with postoperative ASD were matched to control patients without ASD that had similar surgical and medical characteristics. Demographic, operative, postoperative, QOL, and cost data were collected for both the ASD and control groups, up to 1-year follow-up.

Results: We reviewed 137 patients that underwent primary lumbar fusion, and identified $13(9 \%)$ who developed ASD. The average 
follow-up for the ASD group was 53.8 months and for the control group was 45.1 months. ASD was defined as radiographic and clinical findings requiring corrective surgery for pathology adjacent to the fusion. No significant differences in demographic or operative characteristics were observed between the ASD and the matched control group. The ASD group had significantly worse EQ-5D Health State scores 6 months following primary fusion and revision operations, compared to controls ( $\mathrm{p}=0.03$ and $\mathrm{p}=0.05$, respectively). Furthermore, the ASD group had worse QALYs 1 year after primary fusion and revision operation compared to controls $(\mathrm{p}=0.002$ and $\mathrm{p}=0.02$, respectively). Patients with ASD also had worse PHQ-9 scores at 6 months following revision operation compared to primary fusion $(\mathrm{p}=0.003)$. With respect to financial costs, the ASD group had significantly higher costs related to hospital admission $(\mathrm{p}<0.0001)$, surgery $(\mathrm{p}<0.0001)$, imaging $(\mathrm{p}=0.02)$, and total direct costs $(\mathrm{p}<0.0001)$ when compared to the control group. There were no significant differences in indirect costs between the two groups.

Conclusions: This study is the first quantification of the impact of ASD on patients' quality of life and associated costs for care. We found that ASD was associated with lower QOL outcomes and a significantly greater overall cost as compared to patients undergoing primary lumbar fusion.

203. The Impact of Dynamic Alignment, Motion and Center of Rotation on Myelopathy Grade and Regional Disability in Cervical Spondylotic Myelopathy

Shian Liu, Renaud Lafage, Justin S. Smith MD PhD (University of Virginia Health System), Themistocles Protopsaltis MD, Virginie Lafage PhD (NYU Langone Medical Center), Vincent Challier, Christopher I. Shaffrey MD FACS (University of Virginia), Han Jo Kim MD, Paul M. Arnold MD (Department of Neurosurgery), Jens Chapman MD (University of Washington), Frank Schwab MD, PhD, Eric M. Massicotte MD (Toronto Western Hospital), Tim Yoon, Michael G. Fehlings MD PhD FRCSC FACS (Toronto Western Hospital), Christopher P. Ames MD (University of California San Francisco Neuro Surgery), Justin K Scheer BS (University of California, San Diego)

Introduction: Cervical stenosis is a defining feature of cervical spondylotic myelopathy (CSM). Matsunaga et al proposed that elements of stenosis are both static and dynamic, where the dynamic elements magnify the canal deformation of the static state. We hypothesize that dynamic motion may be associated with myelopathy severity and neck disability and present novel methods of dynamic motion analysis in CSM.

Methods: Post-hoc analysis of a prospective, multicenter database of patients with CSM. 110 patients (34\%) met inclusion criteria: symptomatic CSM, age over 18, baseline flexion/extension radiographs, and health related quality of life (HRQOL) measurements (mJOA, NDI, SF-36, and Nurick grade). The mean age was 57+12 years with $41 \%$ female $(n=46)$. Correlations with HRQOLs were analyzed for regional and focal parameters in flexion and extension. Baseline dynamic parameters $[\mathrm{F} / \mathrm{E}$ cone relative to a fixed $\mathrm{C} 7$, center of rotation (COR), range of motion (ROM) arc relative to the COR] were also analyzed for correlations to HRQOLs.

Results: At baseline, the mean HRQOLs demonstrated disability and the mean radiographic parameters demonstrated sagittal malalignment. Among regional parameters, there was a significant correlation between increased $\mathrm{C} 2-\mathrm{C} 7$ angle in flexion and Nurick grade $(\mathrm{R}=0.189, \mathrm{p} 0.048)$ with no significant correlations in extension. Focal parameters including C7 slip were significantly correlated with disability (Flex $\mathrm{R}=-0.377, \mathrm{p}$ 0.003; Ext $\mathrm{R}=-0.261, \mathrm{p}$ 0.027 ). Reduced flexion/extension motion cones, a more posterior center of rotation, and smaller range of motion correlated with worse HRQOLs.

Conclusions: Dynamic motion analysis may play an important role in understanding CSM. Focal parameters demonstrated a sig- nificant correlation with worse HRQOLs especially C7 slip in flexion and extension. Novel methods of motion analysis demonstrated reduced motion cones correlated with worse myelopathy grades. More posterior center of rotation and smaller range of motion were both correlated with worse general health scores (PCS \& Nurick).

204. Comparative Sensitivity of Intraoperative Neuromonitoring with MEP in Predicting Post-operative Neurological Deficits: Non-degenerative versus Degenerative Myelopathy

Aaron J. Clark MD PhD (Semmes-Murphey Neurologic \& Spine Institute), Michael Safaee MD (University of California, San Francisco), Dean Chou M.D. (University of California San Francisco), Philip R. Weinstein MD (University of California, San Francisco), Annette Molinaro MA PhD, John P Clark III PhD (UCSF), Praveen V. Mummaneni MD (University of California San Francisco Spine Center)

Introduction: The use of intraoperative neurophysiological monitoring $(\mathrm{IONM})$ in spine surgery may assist the surgeon in taking corrective measures to prevent postoperative neurological deficits, but the efficacy of monitoring in patients with myelopathy from non-degenerative causes has not been quantified. We compared the sensitivity and positive predictive value(PPV) of IONM in patients with myelopathy caused by non-degenerative processes to those with degenerative cervicothoracic spondylotic myelopathy(CSM).

Methods: We retrospectively reviewed our cervical myelopathy surgical cases during a one-year period to identify patients with CSM and non-degenerative myelopathy. Data on intraoperative motor evoked potential(MEP) changes and postoperative new deficits were collected from patient charts. Categorical variables were analyzed by Fishers exact test. Receiver-operator curves assessed MEP performance in the two groups.

Results: 144 patients were identified. 102 patients had degenerative CSM and 42 had non-degenerative myelopathy(24 extra-axial tumors, 12 infectious processes, 5 traumatic fractures, and 1 rheumatoid arthritis). In the CSM group, there were $8 \mathrm{MEP}$ alerts and 5 new deficits. There was a significant association between MEP alerts and post-operative deficits $(\mathrm{p}<0.001)$. The corresponding sensitivity was $71 \%$, the specificity was $94 \%$, the PPV was $45 \%$, and the negative predictive value(NPV) was $98 \%$. In the nondegenerative group, there were $10 \mathrm{MEP}$ alerts and 1 new deficit. In this non-degenerative group, there was no association between MEP alerts and new deficits $(\mathrm{p}=1.0)$. The sensitivity(33\%), specificity $(74 \%)$, and PPV( $9 \%$ ) were lower. Among patients with CSM, the model performed well for predicting post-operative deficits with an area under the curve(AUC) of 0.826 . This appeared better than the non-degenerative group, although did not reach significance(AUC $0.538, \mathrm{p}=0.16$ )

Conclusions: Based on this large retrospective analysis, intraoperative MEP in surgery for non-degenerative myelopathy appears to be less sensitive to cord injury and less predictive of postoperative deficits when compared to CSM cases.

205. Clinical and Surgical Predictors of Perioperative Complications in Patients with Degenerative Cervical Myelopathy: Results from the Multicenter, Prospective AOSpine International Study on 479 Patients

Michael G. Fehlings MD PhD FRCSC FACS (Toronto Western Hospital), Lindsay Tetreault Bsc, Branko Kopjar, Gamaliel Tan MD (Tan Tock Seng Hospital), Pierre Cote DC PhD, Helton L A Defino PhD (Faculdade de Medicina de Ribeirão Preto), Shashank Sharad Kale MBBS MS MCh MD (All India Inst of Med Sciences), Giuseppe Barbagallo MD (Policlinico University Hospital), Ronald HMA Bartels MD PhD (Radboud University Medical Center), Qiang Zhou MD (Southwestern Hospital), Paul M. Arnold MD 


\section{Meeting Abstracts: AANS/CNS Section on Disorders of the Spine and Peripheral Nerves}

(Department of Neurosurgery), Mehmet Zileli MD (1416 sok 7), Yasutsugu Yukawa MD, Osmar Moraes MD, Massimo Scerrati MD (Via Conca 71), Tomoaki Toyone MD PhD (Teikyo University Chiba Medical Center), Masato Tanaka MD, Ciaran Bolger MD (Frenchay), Manuel Alvarado MD (Hospital San Juan de Dios)

Introduction: Surgery for the treatment of cervical spondylotic myelopathy (CSM) is not without associated morbidity and is typically accompanied by complication rates between 11 and $38 \%$. By identifying important clinical and surgical predictors of complication development, clinicians can recognize their high-risk patients and institute appropriate prevention plans. This study aims to identify important clinical and surgical predictors of perioperative complications in patients with CSM.

Methods: Four-hundred and seventy-nine surgical CSM were enrolled in the prospective CSM-International study at sixteen global sites. A panel of physicians reviewed all adverse events and classified each one as related or unrelated to surgery. Univariate analyses were performed to determine demographic and surgical differences between patients who suffered a perioperative complication and those who did not. A complication clinical prediction rule was developed using multiple logistic regression.

Results: Eighty patients experienced 92 perioperative complications $(16.7 \%)$. Univariately, the major clinical risk factors were OPLL $(\mathrm{p}=0.022)$, the number of comorbidities $(\mathrm{p}=0.020)$, diabetes $(\mathrm{p}=0.004)$, and co-existing gastrointestinal disorders $(\mathrm{p}=0.045)$. Patients undergoing a 2-stage surgery and those with a longer operative duration were also at a greater risk of perioperative complications. A final model consisted of diabetes $(\mathrm{OR}=2.35, \mathrm{p}=0.039)$, age $(\mathrm{OR}=1.02,0.25)$, operative duration $(\mathrm{OR}=1.003, \mathrm{p}=0.17)$, two stage surgery $(\mathrm{OR}=20.37, \mathrm{p}=0.012)$, OPLL $(\mathrm{OR}=1.82, \mathrm{p}=0.064)$, gastrointestinal co-morbidities $(\mathrm{OR}=2.53, \mathrm{p}=0.020)$ and $\mathrm{BMI}$ $(\mathrm{OR}=1.06, \mathrm{p}=0.10)$.

Conclusions: Patients are at a higher risk of perioperative complications if they are older; have OPLL, a higher BMI, diabetes or gastrointestinal disorders; and if they undergo a two-stage surgery and a long operation.

\section{Association Between Surgeon Experience and Complication Rates in Adult Scoliosis Surgery: A Review of 5,699 Cases from the Scoliosis Research Society Database 2004-2007}

Branko Skovrlj MD (Mount Sinai School of Medicine), John M. Caridi MD (Mount Sinai Neurosurgery), Samuel K Cho

Introduction: The complexity of adult deformity surgery (ADS) in the older population has been associated with increased complication rates. Multiple patient- and surgery-related factors have been shown to increase the risk of complications in ADS. No study exist evaluating surgeon experience as a risk factor for complications in ADS. The purpose of this study was to evaluate whether surgeon experience is associated with complications rates in ADS.

Methods: The Scoliosis Research Society Morbidity and Mortality database was queried for patients aged $=18$ years who underwent ADS from 2004-2007. Patient demographics, surgical characteristics, complications and surgeon membership status were analyzed. Two-tailed t-test and chi-square tests were performed with $\mathrm{p}<0.05$ considered significant.

Results: A total of 5,699 patients underwent ADS. The average patient age was 48.3 years. Patients operated by candidate members were older than those operated by active members ( 49.3 versus 48.0 , $\mathrm{p}=0.048)$. Active members performed 4,260 (74.7\%) operations while candidate members performed $1,439(25.3 \%)$ operations. Of those, $1,153(20.2 \%)$ were revisions. A total of $707(12.4 \%)$ complications were recorded, $519(12.2 \%)$ for active and $188(13.1 \%)$ for candidate members, respectively $(\mathrm{p}=0.380)$. Mortality rate was $0.26 \%$. Spinal cord complications accounted for $0.61 \%$ of all cases. Active members had 21(0.49\%) spinal cord complications, while candidates had $14(0.98 \%)(\mathrm{p}=0.051)$. There were a total of $178(3.1 \%)$ surgical site infections (SSI). Active members had 82 (1.9\%) deep SSI, while candidate members had 38 (2.6\%) deep SSI $(\mathrm{p}=0.110)$. Active members had $35(0.8 \%)$ superficial SSI while candidate members had $23(1.6 \%)$ superficial SSI $(\mathrm{p}=0.015)$.

Conclusions: There was a statistically significant, two-fold increase in the rate of superficial SSI among candidate members. A statistically insignificant trend towards higher spinal cord complications and deep SSI among candidate members was encountered. From the findings of this study, it appears that surgeon experience was not significantly associated with complications in ADS.

207. Scoliosis Surgery in the Elderly: Complications, Readmissions, Reoperations, and Mortality

Doniel Drazin MD MA, Lutfi Al-Khouja (Cedars-Sinai Spine Center), Beatrice Ugiliweneza MSPH (University of Louisville), Faris Shweikeh BS (Cedars-Sinai Medical Center), J. Patrick Johnson MD (Spine Center), Terrence T. Kim MD, Maxwell Boakye MD

Introduction: The operative management of scoliosis in adults, particularly the elderly, remains controversial. In this study, we evaluated outcomes in elderly patients undergoing surgery for scoliosis.

Methods: Patient data was obtained from a 5\% sample Medicare Provider Analysis and Review (MEDPAR) database. Patients $>65$ years of age diagnosed with scoliosis and receiving lumbar laminectomy or fusion surgery were identified between years 2005-2011.

Results: A total of 453 patients were analyzed (262 between age 66-74y, and $191>75 y$ ). Complication rates, wound infections, repeat surgery at 1-, 2-, 5-years, and 30-day mortality between the age groups did not differ significantly. Ages $66-74 y$ were most commonly discharged home/self-care, while patients $>75 \mathrm{y}$ were discharged to skilled nursing facilities $(38.55 \%$ vs. $34.04 \%, \mathrm{p}$-value $=$ 0.0011 ). Additionally, $9.92 \%$ of the $66-74 y$ group were re-admitted within 30-days compared to $17.28 \%$ in those $75 y+(p-$ value $=$ $0.0217)$. By 30 days post-discharge, the complication rate for the younger group increased by $2.67 \%$ compared to $9.37 \%$ in the older group ( $20.99 \%$ vs. $26.65 \%$, respectively).

Conclusions: There was no statistical significance in the rate of complications and wound infections between the two age groups analyzed. Although there was a trend towards higher 30-day mortality in those $75 y+$, and increased reoperation rates in $66-74 y$, this did not achieve statistical significance. An increased rate of readmission and non-routine discharges was evident in those $75 y+$. These findings suggest differential outcomes to scoliosis surgery in the elderly population stratified by age. Future prospective studies are warranted to understand risk factors and other confounding variables that influence outcome.

208. Comparison of Resource Use and Health-related Qualityof-life Outcomes Across Multiple Centers Performing Adult Spinal Deformity Surgeries

Richard A. Hostin MD (Southwest Scoliosis Institute / Baylor Scoliosis Center), Chessie Robinson MA (4708 Alliance Blvd), Michael F. O'Brien MD, Christopher P. Ames MD (University of California San Francisco Neuro Surgery), Virginie Lafage PhD (NYU Langone Medical Center), Frank Schwab MD, PhD, Munish Gupta MD (University of California - Davis; Ortho Surgery), Justin S. Smith MD PhD (University of Virginia Health System), Douglas C. Burton MD, Shay Bess MD (Rocky Mountain Scoliosis and Spine Center), Christopher I. Shaffrey MD FACS (University of Virginia), Ian McCarthy PhD (4708 Alliance Blvd.)

Introduction: Little is currently known regarding the variation in resources used and the associated outcomes in complex spine 
surgeries. Our study examines multi-center variability in patientlevel surgical resource use alongside health-related quality-of-life (HRQoL) improvements following adult spinal deformity (ASD) surgery.

Methods: This is a retrospective analysis of a multi-center prospective database of consecutive ASD patients. HRQoL outcomes were calculated from the ODI, SRS-22 and SF-36 domain scores. Changes in HRQoL are estimated as the difference between baseline and 2-year values. Patient-level surgical resources include blood use, bone morphogenetic protein (BMP) volume, length of stay and implants. Patients were classified by sagittal modifier (mild, moderate, or severe) and analyzed across centers. Statistical analysis was performed using analysis of variance and multivariable regression methods comparing HRQoL outcomes and resource use across center, considering the overall patient cohort and each modifier group.

Results: Baseline and 2-year HRQoL data were available for 226 surgical ASD patients, with an average age of $56(18-84)$ who were predominantly female $(\mathrm{N}=189,84 \%)$. Significant differences were found in the average 2-year change in HRQoL across centers, however this difference becomes insignificant after controlling for patients within the same modifier groups $(p>.05)$. Significant differences were found across centers in average resources used per surgery $(p<0.05$. Significant differences were found in average BMP and screw use across all modifier groups. After accounting for clinical, demographic, and regional characteristics at the patient level, variation among centers persisted in both screw use and total BMP volume with no corresponding statistical differences in HRQoL outcomes.

Conclusions: The use of additional surgical resources does not appear to impact 2-year HRQoL outcomes following surgery for ASD. In order to improve efficiency in ASD surgery, standardization of physician practice patterns and resource use could help curb costs without negatively impacting patient HRQoL.

\section{Regional and Global Correction Loss in Long Adult Deformity Constructs: Magnitude, Location, and Factors from 183 Patients with 2 Year Follow-up}

Justin K Scheer BS (University of California, San Diego), Virginie Lafage PhD (NYU Langone Medical Center), Michael P Kelly MD (Washington University), Richard A. Hostin MD (Southwest Scoliosis Institute / Baylor Scoliosis Center), Robert Hart MD (Ohsu Spine Center), Eric Klineberg MD (University of California - Davis), Themistocles Protopsaltis MD, Vedat Deviren MD (Univeristy of California San Francisco), Daniel M. Sciubba BS MD (Johns Hopkins University), Shay Bess MD (Rocky Mountain Scoliosis and Spine Center), Christopher I. Shaffrey MD FACS (University of Virginia), Frank Schwab MD, PhD, Justin S. Smith MD PhD (University of Virginia Health System), Christopher P. Ames MD (University of California San Francisco Neuro Surgery)

Introduction: Adult deformity patients(pts) can lose spino-pelvic correction over time, especially in the sagittal plane. The goal of this study was to quantitate this loss in verses unfused segments. Variables examined include presence of interbody fusion, rod diameter, rod material, age and preop sagittal alignment.

Methods: A retrospective review of a multicenter, prospective ASD database was conducted. Inclusion criteria: age $=18 \mathrm{yr}$, ASD, no revisions between $>6 \mathrm{wk}$ and $<2 \mathrm{yrs}$ postop. Spinal pelvic parameters, thoracic kyphosis (TK:T2-T12) and lumbar lordosis (LL:L1-S1) were measured overall and within and outside of the instrumented segments. Changes for SVA, PT, PI-LL, TK, and LL between $6 \mathrm{wks}-2 \mathrm{yrs}$ postop were calculated. Of these pts, the amount of thoracic loss and TL loss within and outside of the instrumented segments was also determined as well as a percentage of the total loss for each region.

Results: 183 pts met criteria. Between $6 \mathrm{wk}-2 \mathrm{yr}$ postop $61.7 \%$ of pts lost SVA $(4.4 \pm 4.3 \mathrm{~cm}), 65.0 \%$ lost PT $(4.7 \pm 4.0 \mathrm{deg})$, and
40.4\% lost PI-LL (16.2 $\pm 9.3 \mathrm{deg}) .63 .9 \%$ had TH loss $(8.0 \pm 8.9 \mathrm{deg})$ and $56.8 \%$ had LL loss $(5.8 \pm 4.5 \mathrm{deg}) .41 .5 \%$ lost $>2 \mathrm{~cm}$ SVA. Of the pts with $>2 \mathrm{~cm}$ SVA loss, $60.5 \%$ had LL loss, and of those, $82.6 \%$ was within instrumented segments. Of the pts with LL loss $13.8 \%$ had $>10 d e g$ loss. Pts with an interbody fusion had a $2.3 \mathrm{x}$ $(\mathrm{p}=0.0076)$ likelihood of having any LL loss but interbody fusion was protective against $>10 \mathrm{deg}$ change $(7.1 \%)$. Pts $=65$ yrs old were $9.4 \mathrm{x}(\mathrm{p}<0.0001)$ more likely to lose LL than pts $=45 \mathrm{yrs}$ old $(4.4 \%)$. Pts with preop C7 SVA $>5 \mathrm{~cm}$ were $2.4 \mathrm{x}(\mathrm{p}=0.0035)$ more likely to lose LL.

Conclusions: Loss of initial regional and global sagittal correction is common in ASD surgery and also occurs inside the instrumented segments. Older age and preop sagittal deformity are risk factors for overall loss of correction. Interbody fusion was only protective for LL loss $>10 \mathrm{deg}$.

\section{Complications of the Minimally Invasive Anterior Column Release after Restoration of Global Sagittal Balance in Adult Deformity Surgery}

Gisela Murray MD, Juan S. Uribe MD (University of South Florida - Division of Neurosurgery), Konrad Bach MD (University of South Florida), Joshua Marshall Beckman MD (University of South Florida)

Introduction: Minimally Invasive techniques are taking a larger role in deformity surgery. Lateral retroperitoneal transpsoas anterior column release (MIS-ACR) is a newer minimally invasive alternative to posterior osteotomy techniques in correcting and promoting lordosis. Moreover, this procedure avoids potential complications related to conventional osteotomies

Methods: A retrospective study was performed of all patients who underwent MIS-ACR in our institution since 2010. This study evaluates the development of perioperative and postoperative complications in the setting of adult spinal deformity (ASD) correction surgery, specifically ones related to the MIS-ACR lateral transpsoas technique

Results: There were a total 31 patients who underwent $47 \mathrm{MI}$ ACRs. The mean age of the cohort was 62. Mean follow up was 11 months (1-38 months). The average change lumbar lordosis (LL) was 20 degrees and in sagittal vertical axis (SVA) was $5.4 \mathrm{~cm}$. Of 47 MIS-ACR procedures, the total of major complications related to the ACR procedure itself were $10(21 \%)$; 9 in the perioperative period and 1 found at the postoperative period. Of these, iliopsoas weakness was seen in 8 patients. At follow up, only 1 patient remained with mild motor deficit. One patient developed retrograde ejaculation. The average EBL per level was $56 \mathrm{ml}$. No revision surgery needed related to anterolateral approach. Furthermore, there were no vascular, or visceral complications associated with the MIS-ACR

Conclusions: The MIS-ACR is the maximum sophistication of the minimal invasive lateral transpsoas approach. This procedure has the advantage of improving spinal global alignment on ASD surgeries. Most importantly, this study gives the impression that MIS-ACR is associated with fewer complications and lower blood loss compared to conventional posterior osteotomies. Given that in our experience most of the complications were transient, MIS-ACR has demonstrated to be a reproducible and safe technique that warrants a major role in the correction of adult deformity. 


\section{Meeting Abstracts: AANS/CNS Section on Disorders of the Spine and Peripheral Nerves}

\section{Re-operation Rates in Minimally Invasive, Hybrid and Open Surgical Treatment for Adult Spinal Deformity with Minimum 2-Year Follow-up}

Kai-Ming G.Fu MD PhD (Weill Cornell Medical College), Adam S. Kanter MD (University of Pittsburgh Medical Center Presbyterian), David O. Okonkwo MD PhD (University of Pittsburgh), Gregory Mundis MD (San Diego Center for Spinal Disorders), Praveen V. Mummaneni MD (University of California San Francisco Spine Center), Neel Anand MD, Richard G. Fessler MD, Paul Park MD (University Of Michigan, Department of Neurosurgery), Frank LaMarca MD, Juan S. Uribe MD (University of South Florida Division of Neurosurgery), Michael Y. Wang MD FACS (University of Miami)

Introduction: Minimally invasive surgical (MIS) techniques are gaining popularity in the treatment of ASD with the premise of equivalency in outcomes and complication reduction. Potential limitations to MIS techniques are decreased corrective capacity, concern for long-term efficacy, and potential need for revision surgery. The current study aims to compare re-operation rate and indications following MIS, hybrid and open surgery for ASD.

Methods: Two multi-center databases were retrospectively analyzed . Inclusion criteria: age $>18$ years with minimum $20^{\circ}$ coronal lumbar Cobb, minimum 3 levels fused and 2 year follow-up. Patients were propensity matched for preop SVA, PI-LL, and levels fused, resulting in 114 patients in three subgroups of 38 patients: (1) MIS: lateral or transforaminal lumbar interbody fusion (LIF) and percutaneous pedicle instrumentation, (2) Hybrid: MIS LIF with open posterior segmental fixation (PSF), and (3) Open: PSF +/- osteotomies.

Results: There were no significant differences between groups in pre-op SVA or PI-LL ( $p>0.05)$, however the MIS group had significantly fewer levels fused (4.7) than the open group (6.8) $(\mathrm{p}=0.002)$. The rate of revision surgery was not significantly different between the groups $(\mathrm{p}=0.196)$ : MIS=15.8\% (6/38), Hybrid=31.6\% (12/38), Open $=31.6 \%(12 / 38)$. The most common reason for reoperation in the Open group was neuro deficit (10.5\%) followed by PJK $(7.9 \%)$. The most common reason in the Hybrid group was PJK $(13.2 \%)$ followed by infection (7.9\%). The most common reason in the MIS group was pseudarthrosis (7.9\%).

Conclusions: Re-operation rates were not statistically different between the MIS, Hybrid, and Open surgical groups, however the incidence was twice as high in Hybrid and Open groups. The most common reasons for reoperation were PJK, neuro deficit, and infection for the hybrid and open groups, but pseudarthrosis in the MIS group.

\section{Simultaneous Lateral Interbody Fusion and Posterior} Percutaneous Instrumentation: A Multi-center Analysis

Doniel Drazin MD MA, Terrence T. Kim MD, Faris Shweikeh BS (Cedars-Sinai Medical Center), Luis Marchi MSc (Instituto de Patologia da Coluna), Jonathan N Sembrano MD (University of MInnesota), Joseph R O'Brien MD MPH (George Washington University MFA), Luiz Pimenta MD (Instituto de Patologia da Coluna), J. Patrick Johnson MD (Spine Center)

Introduction: Lateral interbody lumbar fusion with posterior instrumentation is becoming more common and is typically performed by repositioning the patient to complete the circumferential procedure. In this retrospective multi-center study, the authors describe techniques and results from patients in which both procedures were performed simultaneously from a single lateral position without repositioning.

Methods: Results were analyzed from 40 patients at 4 different institutions who underwent single lateral-posterior position surgery. Patient diagnoses included: pars fractures with instability, grade two spondylolisthesis, symptomatic degenerative disk disease. Indications for surgery included worsening back pain with and without radiculopathy in patients who failed exhaustive conservative management. Exclusion criteria: "small or atrophic/dystrophic" pedicles, "rotated, asymmetric" pedicles, certain L5 pedicles difficult to visualize. Technique was not performed beyond two levels of instrumentation. The technique requires placing the patient in a specific lateral position on a radiolucent table. The ideal operating table has multi-plane adjustment capabilities (Sliding Skytron, breakable Jackson table). After performing standard transpsoas lateral interbody fusion, the patient's position remains unchanged for the posterior percutaneous instrumentation/fusion.

Results: In patients undergoing both procedures simultaneously without repositioning, operative time from incision to closure was $155.4 \pm 53.5$ minutes on average with intraoperative blood loss of $80.5 \pm 59.1 \mathrm{ml}$. Non-repositioned patients were hospitalized an average of $3.03 \pm 1.2$ days. At mean follow-up of $10.7 \pm 5.5$ months, $93.8 \%$ of patients were fused. Complications included 2 reoperations $(5.0 \%), 2$ transient lower extremity weakness $(5.0 \%)$, 2 transient lower extremity paresthesia (5.0\%).

Conclusions: Lateral interbody fusion and percutaneous posterior pedicle instrumentation can be accomplished in a single lateral decubitus position in select patients with adequately sized pedicles, offering this advantage over sequential surgery requiring repositioning: a faster operative time. Although a 5\% transient neurologic complication rate was noted, overall patient outcomes were excellent and comparable to procedures done in previous series.

\section{Rate of Radiographic Appearance of Fusion and Vertebral Body Fracture Following Lateral Interbody Instrumentation}

Timothy C. Ryken MD MS FACS (Iowa Brain \& Spine Institute), Steven Dillavou MS PA-C (Iowa Spine \& Brain Institute), Laura Chapin PhD, Tia Derflinger, Kelly Ward

Introduction: Over a four-year period, 79 patients underwent 153 levels of stand-alone lateral interbody instrumentation by a single spine surgical team. The objective of this retrospective analysis is to further investigate the ability to achieve fusion, as well as the potential of vertebral body fractures occurring adjacent to an operative level.

Methods: This adult population included 42 females and 37 males with ages ranging from 21 to 88 years. This included 45 right and 34 left lateral approaches. Radiographic images including standing $\mathrm{x}$-rays and standard CT were collected and analyzed for appearance of fusion and for any vertebral body fracture adjacent to an operative level.

Results: Of the 79 patients who underwent lateral interbody instrumentation, 61 had images available at least one year postoperatively or had likely or solid radiographic evidence of fusion up to the point of analysis. Eleven of these appeared to have solid fusion, 44 appeared to have likely progress toward solid fusion, and 6 had questionable or no evidence of fusion. During this series, 12 patients had some degree of vertebral body fracture or settling adjacent to an operative level. This represented a total of 12 within the group of operative levels. Of these 12 patients, 2 had compression fractures. One underwent vertebroplasy and the other underwent posterior instrumentation. Eleven of the 12 patients later went on to demonstrate evidence of likely progress toward solid fusion or solid fusion. Additionally, one patient required supplemental posterior instrumentation to later achieve solid fusion.

Conclusions: In this series, radiographic evidence of solid fusion occurred in relatively few (18\%) of patients. The occurrence of a fracture or settling adjacent to an implant was relatively common $(19.7 \%)$. These may be related to choice of implant height, graft choice, or excessive lateral bending in positioning. 


\section{Short-segment Percutaneous Pedicle Screw Fixation with} Cement Augmentation for Tumor-induced Spinal Instability

Nelson Moussazadeh MD (New York Presbyterian Hospital (Cornell Campus)), David Rubin MD (Legacy Spine \& Neuurological Specialists), Lily McLaughlin, Eric Lis, Mark H. Bilsky MD (Memorial Sloan Kettering Cancer Center), Ilya Laufer MD (Memorial Sloan-Kettering Cancer Center)

Introduction: Pathologic vertebral compression fractures (VCF) represent a major source of morbidity and diminished quality of life in the spinal oncology population. Procedures with low morbidity that effectively treat patients with pathologic fractures are especially important in the cancer population where life expectancy is limited. Vertebroplasty and kyphoplasty are often not effective for mechanically unstable pathologic fractures extending into the pedicle and facet joints. Combination of cement augmentation and percutaneous instrumented stabilization represent a minimally invasive treatment option that does not delay radiation and systemic therapy. In this study we evaluated the safety and efficacy of cement-augmented short-segment percutaneous posterolateral instrumentation for tumor-associated VCF with pedicle and joint involvement.

Methods: Forty-four consecutive patients underwent cementaugmented percutaneous spinal fixation for unstable tumors between 2011 and 2014. Retrospective analysis of prospectively-collected data, including visual analog pain response score (VAS) and procedural complications, was performed.

Results: Patients with a median composite Spinal Instability Neoplastic Score of 10 (range=8-15) were treated with constructs spanning 1-4 disk spaces (median 2 spaces, constituting $84 \%$ of all cases). The proportion of patients suffering severe pain decreased from $86 \%$ preoperatively to $0 \%$; $65 \%$ of patients reported no referable instability pain postoperatively. There was one adjacent level fracture responsive to kyphoplasty, and one case of asymptomatic screw pull-out. Two patients subsequently required decompression in the setting of disease progression despite radiation; there was no peri-operative morbidity.

Conclusions: Percutaneous cement-augmented posterolateral spinal fixation is a safe and effective option for palliation of appropriately-selected mechanically-unstable VCF that extend into pedicle and/or joint.

\section{Assessing the Impact of Thoracolumbar Deformity on Cervical Disability and Pain: How the Back Affects the Neck}

Aaron J. Clark MD PhD (Semmes-Murphey Neurologic \& Spine Institute), Taemin Oh BA (UCLA David Geffen School of Medicine), Rajiv Saigal MD PhD, Carol MY Li (University of California San Francisco), Devon J Ryan BA (NYU Hospital for Joint Diseases), Eric Klineberg MD (University of California - Davis), Robert Hart MD (Ohsu Spine Center), Han Jo Kim MD, Frank Schwab $M D, P h D$, Justin S. Smith MD PhD (University of Virginia Health System), Themistocles Protopsaltis MD, Virginie Lafage PhD (NYU Langone Medical Center), Christopher P. Ames MD (University of California San Francisco Neuro Surgery), Justin K Scheer BS (University of California, San Diego)

Introduction: Patients (pts) with thoracolumbar deformity employ compensatory mechanisms to maintain horizontal gaze, which may result in cervical mal-alignment. However, the effects of these reciprocal changes on regional cervical disability have not been well studied in this pt population. The objective of the present study is two-fold: a) to report baseline regional neck disability in adult spinal deformity (ASD) and b) to define the relationship between neck disability and degree of cervical deformity.

Methods: This is a single center retrospective analysis of consecutive ASD pts. Pre-op radiographs were analyzed for cervical, thoracic, lumbar, and spino-pelvic parameters. Pre-op disability was assessed using indices for neck disability (NDI), Oswestry disability (ODI), neck pain (VAS-neck), and back pain (VAS-back). Pts were also grouped into mild sagittal plane deformity $(\mathrm{C} 7 \mathrm{SVA}<5 \mathrm{~cm}$ ) and severe $(>10 \mathrm{~cm})$. Correlations were made by linear regression, and comparisons between groups were made using student's t-test.

Results: 61 pts were assessed. Mean preop disability indices were: $\mathrm{NDI}=32$, ODI=49, VAS-neck=3.2, and VAS-back=6.9. Significant correlations were found between: ODI and NDI $(\mathrm{p}<0.001, \mathrm{R}=0.61)$, ODI and VAS-back $(\mathrm{p}<0.001, \mathrm{R}=0.68)$, NDI and VAS-back $(\mathrm{p}=0.004, \mathrm{R}=0.39)$, and NDI and VAS-neck $(\mathrm{p}<0.001, \mathrm{R}=0.61)$. There were significant correlations between ODI and C7 sagittal vertical axis (SVA; $\mathrm{p}=0.005)$, pelvic tilt $(\mathrm{p}=0.004)$, and pelvic incidence/lumbar lordosis mismatch $(\mathrm{p}=0.03)$. No other significant correlations were found between disability indices and radiographic parameters. No differences were found between pts with mild or severe sagittal deformity in NDI (31.8 vs 31.5 , respectively; $p=0.96$ ) or VAS-neck (3.1 vs 3.3, respectively; $\mathrm{p}=0.80$ ).

Conclusions: Thoracolumbar deformity pts have moderate neck disability (NDI) and mild-moderate neck pain (VAS) preoperatively. There is a correlation between neck and low back regional disability, and NDI was highly correlated to back and neck pain. However, neither cervical disability nor severe sagittal deformity appears to correlate with neck disability or pain.

216. Adult Spinal Deformity Patients with Concomitant Preoperative Cervical Deformity Have Inferior Outcomes at 2 Year Follow up

Justin K Scheer BS (University of California, San Diego), Justin S. Smith MD PhD (University of Virginia Health System), Peter $G$ Passias MD, Alex Soroceanu, Anthony Boniello, Frank Schwab MD, PhD, Christopher I. Shaffrey MD FACS (University of Virginia), Han Jo Kim MD, Themistocles Protopsaltis MD, Gregory Mundis MD (San Diego Center for Spinal Disorders), Munish Gupta MD (University of California - Davis; Ortho Surgery), Eric Klineberg $M D$ (University of California - Davis), Virginie Lafage PhD (NYU Langone Medical Center), Christopher P. Ames MD (University of California San Francisco Neuro Surgery)

Introduction: A high prevalence of cervical deformity (CD) has been identified among adult spinal deformity (ASD) patients undergoing surgical treatment. The clinical impact of this is uncertain. This study aims to quantify the difference in patient-reported outcomes among ASD patients based on presence of $\mathrm{CD}$ prior to treatment.

Methods: Retrospective review of a multicenter prospective database of surgical ASD patients with 2-year follow-up. CD was defined as: C2C7 SVA $>4 \mathrm{~cm}$, cervical kyphosis (CK) C2C7angle $>0$. Univariate testing was performed using t-tests, or tests of proportion. Multivariate models determined impact of pre-op $\mathrm{CD}$ on Health Related Quality of Life (HRQL) scores (SF-36, ODI, SRS) and obtaining Minimally Clinically Important Difference (MCID) at 2 years.

Results: 191 patients met criteria, of which 65 with pre-op C2C7 SVA $>4 \mathrm{~cm}, 57$ with CK. Patients with and without CD saw improvements in 2-year HRQL scores $(\mathrm{p}<0.001)$. Overall, patients with pre-op CD had inferior post-op HRQL and were less likely to achieve MCID for HRQL at 2-years. Those with pre-op SVA $>4 \mathrm{~cm}$ had worse ODI, PCS, SRS activity, appearance, pain, and total, and were less likely to meet MCID for ODI, PCS, SRS activity and pain scores (odds ratio 0.39 for ODI CI 0.19:0.81 $\mathrm{p}<0.05),(0.34$ for PCS CI 0.16:0.72 p<0.05), (0.29 CI 0.14:0.62 p<0.05 SRS activity) (0.41 CI 0.20:0.86 p<0.05 SRS pain). Similarly, patients with CK had inferior 2-year HRQL scores. Patients without any CD were 4x more likely to reach 2-year SRS activity MCID (OR $0.40 \mathrm{CI}$ 0.19:0.86 p<0.05).

Conclusions: Despite experiencing significant improvements in HRQL scores, pre-op CD in ASD patients is a strong predictor 


\section{Meeting Abstracts: AANS/CNS Section on Disorders of the Spine and Peripheral Nerves}

of inferior outcomes and failure to reach MCID at 2 year follow up. This information will help surgeons educate patients at risk for inferior outcomes and direct future research to improve patient outcomes.

\section{Impact of Regional and Focal Cervical Alignment on Myelopathy Severity: Report of 151 Patients}

Themistocles Protopsaltis MD, Michael G. Fehlings MD PhD FRCSC FACS (Toronto Western Hospital), Shian Liu, Justin S. Smith MD PhD (University of Virginia Health System), Virginie Lafage PhD (NYU Langone Medical Center), Christopher I. Shaffrey MD FACS (University of Virginia), Han Jo Kim MD, Paul M. Arnold MD (Department of Neurosurgery), Jens Chapman MD (University of Washington), Renaud Lafage, Vincent Challier, Frank Schwab MD, PhD, Eric M. Massicotte MD (Toronto Western Hospital), Tim Yoon, Christopher P. Ames MD (University of California San Francisco Neuro Surgery), Justin K Scheer BS (University of California, San Diego)

Introduction: Cervical sagittal alignment (C2-C7 SVA) has been correlated to myelopathy severity but kyphosis as a regional parameter has not been demonstrated to correlate with health related quality of life (HRQOL) measures. We hypothesized that regional and focal sagittal cervical alignment may also correlate with HRQOLs in cervical spondylotic myelopathy (CSM) patients.

Methods: Post-hoc analysis of a prospective, multicenter database of CSM patients. 151 patients met inclusion criteria (47\%): symptomatic CSM, age $>18$, baseline lateral radiographs, modified Japanese Orthopaedic Association (mJOA) and neck disability index (NDI). Average age was $56.3+11.7$ yrs with females making up $43.9 \%(n=69)$ of the study population. Regional alignment and HRQOLs were analyzed with patients stratified into a high cSVA group (hiSVA, $n=45,0.5 \mathrm{SD}>$ mean) or a low cervical sagittal vertical axis (cSVA) group (loSVA, $n=43,0.5 \mathrm{SD}<$ mean). Focal alignment (kyphosis and olisthesis between adjacent vertebrae) analysis was also conducted.

Results: The hiSVA (mean cSVA $39.4+7.4 \mathrm{~mm}$ ) cohort had a significantly worse mJOA than the loSVA (mean cSVA $11.31+5.3 \mathrm{~mm}$ ) cohort (mean mJOA 12.5 vs 13.7, $\mathrm{p}=0.037$ ). No significant correlations between $\mathrm{C} 2-\mathrm{C} 7$ lordosis and HRQOLs existed. There were significant correlations with focal alignment and worse clinical assessments: $\max$ sagittal slip with mJOA $(\mathrm{R}=-0.24$, $\mathrm{p}=0.002)$ and Nurick $(R=0.18, p$ 0.024). Level of slip was also significant, with olisthesis at higher levels correlating with worse health status. Number of kyphotic segments also positively correlated with NDI $(\mathrm{R}=0.19, \mathrm{p}<0.001)$.

Conclusions: Similar to prior studies, high baseline cSVA correlated with significantly worse mJOA. Interestingly, the mean cSVA of the hiSVA cohort was $39.4+7.4 \mathrm{~mm}$, suggesting that $40 \mathrm{~mm}$ may be a potential threshold below which cervical sagittal malalignment should be corrected. Regarding focal alignment, increased olisthesis is correlated with worse mJOA and Nurick grade, especially at higher cervical levels. Even focal abnormalities all along the cervical spine are correlated with worse NDI, demonstrating that while level of olisthesis may matter, the number of vertebrae with a kyphotic relationship may also cause pain and disability.

\section{Frequency of Symptomatic Vertebral Body Compression Fractures Requiring Intervention Following Single Fraction Stereotactic Radiosurgery for Spinal Metastases}

Michael Virk MD PhD (New York-Presbyterian Hospital (Cornell Campus)), James Han (CRB 2), Eric Liss, Yoshiya Josh Yamada MD, FRCP (Memorial Sloan Kettering Cancer Center), Mark $H$. Bilsky MD (Memorial Sloan Kettering Cancer Center), Ilya Laufer MD (Memorial Sloan-Kettering Cancer Center)
Introduction: Single-fraction stereotactic radiosurgery (SRS) 24 Gy dose provides excellent tumor control in patients with spinal metastases. 12-40\% radiographic vertebral compression fracture (VCF) rate has been reported after SRS. No studies have identified the rate of symptomatic fractures. The purpose of this study is to determine the rate of post-SRS symptomatic VCF requiring treatment with kyphoplasty or surgery in the absence of tumor recurrence.

Methods: 336 patients that received single-fraction SRS between C1-L5 were included. Charts and imaging were reviewed for postSRS kyphoplasty or surgery for mechanical instability. Spinal Instability Neoplastic Score (SINS) was calculated for each patient at the time of SRS and at the time of intervention for VCF.

Results: Twenty-six patients $(7.7 \%)$ with thirty-four levels treated with SRS (24Gy) progressed to symptomatic VCF requiring treatment with either kyphoplasty (10) or surgery (16). This represented $45 \mathrm{VCF}$ in 558 levels receiving SRS (8\%). The median time to symptomatic VCF was twenty-seven months. VCF occurred at thirty-two of the thirty-four SRS treated levels in the fracture population, while thirteen VCF occurred adjacent to the level of previous SRS. Median SINS changed from 5 at SRS (1-10) to 11 at stabilization (7-16). Twenty-four patients had biopsy at the time of stabilization that showed no evidence of tumor. The remaining two had no evidence of radiographic or clinical progression. The most common metastatic tumor was renal cell. Median follow up time from date of SRS was thirty-eight months (5-79).

Conclusions: After receiving ablative single-fraction SRS to spinal lesions, $8 \%$ of patients progressed to symptomatic VCF at the treated level in the absence of tumor recurrence. This includes a total of $45 \mathrm{VCF}$, with thirteen occurring at adjacent levels. These results may prove useful in discussions with patients regarding treatment risk and when considering early intervention when imaging evidence of pending VCF is detected.

\section{Novel Osteoinductive Spinal Instrumentation Enhances Osteogenesis and Fusion: A Preliminary Study}

Matthew $R$ MacEwan BSE PhD (Washington University in St. Louis), Michael Talcott DVM, Daniel Moran PhD, Eric C. Leuthardt MD (Washington University School of Medicine)

Introduction: Instrumented spinal fusion continues to exhibit high failure rates in patients undergoing multi-level lumbar fusion, pseudoarthrosis revision, Grade II+ spondolyolithesis or possessing risk factors such as obesity, tobacco use, or metabolic disorders. Direct current (DC) electrical stimulation of bone growth represents a unique surgical adjunct proven to improve fusion success and clinical outcome of vertebral fusion procedures, yet existing spinal fusion stimulators are not optimized to enhance interbody fusion. In an attempt to develop a more advanced method of applying DC electrical stimulation to promote interbody fusion, a novel osteogenic spinal system capable of routing DC current through rigid instrumentation and into the vertebral bodies was fabricated.

Methods: Instrumented single-level posterior lumbar interbody fusion (PLIF) with autologous graft was performed at L4-L5 in adult Toggenburg/Alpine goats using both osteogenic spinal instrumentation (+electrical stimulation) and standard spinal instrumentation (no electrical stimulation). At terminal time points $(3 \mathrm{mo}, 6 \mathrm{mo})$ lumbar spines were explanted for radiographic analysis using a SOMATOM Dual Source Definition CT Scanner and high resolution Microcat II ${ }^{\mathrm{TM}}$ CT Scanner. Examination of trabecular continuity and radiodensity within the fusion mass, along with regional bone formation and osteogenesis, was evaluated to assess success of spinal fusion.

Results: Quantitative analysis of average bone density in pedicle screw beds confirmed that electroactive pedicle screws utilized in the osteogenic spinal system focally enhance bone density in instru- 


\section{Meeting Abstracts: AANS/CNS Section on Disorders of the Spine and Peripheral Nerves}

mented vertebral bodies. Qualitative and quantitative analysis of high-resolution CT scans of explanted lumbar spines further demonstrated that the osteogenic spinal system induced solid bony fusion across the L4-L5 disc space as early as 6 weeks post-operatively. Osteogenic spinal instrumentation demonstrated interbody fusion while standard spinal instrumentation with autograft did not achieve this level of fusion.

Conclusions: Preliminary results demonstrate that novel osteogenic spinal instrumentation accelerates interbody fusion through the focal delivery of DC electrical stimulation. With further technical development and scientific/clinical validation, osteogenic spinal instrumentation may therefore offer an alternative to costly biologic scaffolds and pharmaceutical adjuncts in future spinal fusion procedures.

220. Geriatric Co-Management Reduces Peri-Operative Complications and Shortens Duration of Hospital Stay After Lumbar Spine Surgery: A Prospective Single Institutional Experience

Owoicho Adogwa M.D. M.P.H (Duke University Medical Center, Division of Neurosurgery), Parastou Fatemi, Terence Verla (Duke University School of Medicine), Daniel B Loriaux BS (Duke University Medical Center), Harrison Farber, Gustavo Chagoya BS (Duke University School of Medicine), Jessica Rose Moreno BSN (Duke University Medical Center), Mitchell Heflin, Oren N. Gottfried MD (Duke University Mecical Center), Carlos Antonio Bagley MD (Duke University Medical Center)

Introduction: Older adults undergoing lumbar spine surgery are at risk for adverse outcomes, including delirium, infection, and iatrogenic complications. These complications increase the risk of functional decline, nursing home admission, and death. Here, we assess association of peri-operative co-management by geriatrics and neurosurgery on the incidence of in-hospital complications, length of stay, and readmission after elective lumbar spine surgery.

Methods: In 2013, the Peri-operative Optimization of Senior Health (POSH) program was implemented at Duke University Medical Center with the aim to improve outcomes in patients over 65 years old undergoing lumbar spine fusion surgery. In this model, a geriatrician led team, including a social worker, nurse and nurse practitioner from anesthesia, evaluates patients pre-operatively and follows them daily throughout their hospital stay to manage medical problems and medications, treat pain, prevent delirium, and coordinate rehabilitation, with neurosurgical input. We compared the first 10 cases after the initiation of POSH with the immediately preceding 10 cases on the incidence of peri-operative complications.

Results: Twenty patients undergoing lumbar decompression and fusion were enrolled in this study. Baseline characteristics were similar. Wound infection incidence was lower in the POSH cohort ( $0 \%$ vs. $20 \%$ ). There was significant reduction in the post-operative incidence of urinary tract infections $(20 \%$ vs. $60 \%, \mathrm{p}=0.007)$ and pneumonia ( $0 \%$ vs. $20 \%, \mathrm{p}=0.006)$. Length of in-hospital stay was $37 \%$ lower in the POSH cohort $(6.20 \pm 5.45$ days vs. $9.80 \pm 9.55$ days, $\mathrm{p}=0.045$ ). The POSH cohort had $50 \%$ reduction in discharge to Skilled Nursing Facilities (20\% vs. 40\%). 30-day readmission rates were also significantly lower in the POSH cohort (0\% vs. $30 \%$, $\mathrm{p}=0.080$ ).

Conclusions: In our initial experience, geriatric co-management significantly reduces incidence of post-operative complications, lengths of in-hospital stay, admissions to Skilled Nursing Facilities, and 30-day hospital readmission. Further study could demonstrate which patients benefit the most and which components of the intervention have the greatest impact.

\section{Effects of Psoas Muscle Thickness on Outcomes of Extreme Lateral Interbody Fusion (XLIF) Surgery}

Terence Verla (Duke University School of Medicine), Owoicho Adogwa M.D.M.P.H (Duke University Medical Center, Division of Neurosurgery), Jessica Rose Moreno BSN (Duke University Medical Center), Harrison Farber, Daniel B Loriaux BS (Duke University Medical Center), Oren N. Gottfried MD (Duke University Mecical Center), Joseph S. Cheng MD MS (Vanderbilt University Medical Center), Robert E. Isaacs MD (Duke University Medical Center), Carlos Antonio Bagley MD (Duke University Medical Center)

Introduction: Extreme lateral interbody fusion (XLIF) is one of the major surgical options for the treatment of lumbar pathologies including spondylolisthesis, degenerative disc disease and stenosis. As a result of psoas muscle dissection to gain access to the lumbar spine, muscle-dependent spinal stabilization can be impacted. This study evaluated the effect of the psoas muscle thickness on the incidence of adjacent level degeneration, pseudarthrosis and hardware failure following XLIF surgery.

Methods: A retrospective review of hospital records from 2007 to 2013 was performed of adult patients at our institution undergoing XLIF. Patients $=18$ years who had undergone $=1$ level of XLIF with available pre-operative MRI scans and at least one year follow up were included. Axial psoas muscle thickness was measured at each lumbar intervertebral space. Patients were separated into two cohorts based on the occurrence of complications of interest (adjacent segment degeneration, pseudarthrosis and hardware failure) within one year following surgery. Psoas thickness was assessed based on incidence of complications.

Results: 63 patients were included (Complications: 20; No complications: 43). Demographic characteristics are included in Table 1. There was no significant difference in the median [IQR] number of levels fused between the complications group (2 [1-3] levels) and no-complications group $(2[1-3]), p=0.63$, Table 2 At L1/2 and L5/S1 levels, patients with complications had smaller psoas muscle thickness which trended towards significance $(\mathrm{p}=0.5$ and 0.6 respectively). At L2/3, L3/4 and L4/5 levels, patients with complications had significantly smaller psoas muscle thickness compared to patients without complications $(\mathrm{p}=0.03-\mathrm{L} 2 / 3 ; \mathrm{p}=0.04-\mathrm{L} 3 / 4 ; \mathrm{p}=0.04$ L4/5), Table 3.

Conclusions: This study showed that there is a higher incidence of adjacent segment degeneration, pseudarthrosis and hardware failure in patients with smaller psoas muscle. Given the role of the psoas muscle in spinal stabilization, the effect of psoas muscle on postoperative functional outcomes deserves deeper investigation.

222. XLIF vs. MAS TLIF for the Treatment of Degenerative Spondylolisthesis: Interim Results from an Ongoing Prospective Multi-Center Comparative Study

Robert E. Isaacs MD (Duke University Medical Center), Antoine Tohmeh MD (Northwest Orthopaedic Specialists), Jonathan $N$ Sembrano MD (University of MInnesota)

Introduction: This prospective, multicenter, IRB-approved study aims to compare indirect decompression via XLIF and direct posterior decompression via TLIF for the treatment of low-grade spondylolisthesis with a specific interest in clinical and radiographic results. This abstract serves as an interim report of 6 month outcomes.

Methods: Adult patients with low-grade degenerative spondylolisthesis at 1-2 lumbar levels were treated with either MAS TLIF or XLIF. Motor/sensory evaluations were conducted by the treating physician. Patient-reported outcomes and radiographic measurements were also collected pre- and post-op.

Results: At the time of analysis 52/55 enrolled patients (28 XLIF, 24 TLIF) completed 6 month follow-up, and early post-op neural deficit data was available for 55 patients (29 XLIF, 26 TLIF). Pre- 


\section{Meeting Abstracts: AANS/CNS Section on Disorders of the Spine and Peripheral Nerves}

op neural deficits resolved after surgery in $100 \%$ of XLIF and $73 \%$ of TLIF patients. Mean surgery times favored XLIF (171 min vs $186 \mathrm{~min}) .79 \%$ of XLIF and $27 \%$ of TLIF patients lost less than $100 \mathrm{~mL}$ blood $(\mathrm{p}<0.01)$. Dural tears occurred in 3 TLIF and 0 XLIF patients.. Mild transient post-op hip flexion weakness presented in $34 \%$ of XLIF patients, which all resolved by 6 months. 1/29 XLIF patients had new post-op distal motor weakness, which resolved by 6 months. New post-op sensory function decrease presented in 3/29 XLIF and 2/26 TLIF patients, which all resolved. Post-op VAS and ODI scores were similar between groups, and both showed significant improvement. XLIF resulted in less segmental motion and less early post-op subsidence. Postoperative lumbar lordosis and reduction of spondylolisthesis were similar between treatment groups.

Conclusions: In this early interim analysis comparing XLIF to TLIF for the treatment of low-grade degenerative spondylolisthesis, only subtle differences were identified. Patient-reported pain, function, and satisfaction were comparable. Continued study follow-up is required to make stronger comparisons between procedures.

\section{Measures of Surgical Effectiveness One-Year After Lumbar Spine Surgery Accurately Predict Two-Year Outcomes}

Owoicho Adogwa M.D. M.P.H (Duke University Medical Center, Division of Neurosurgery), Parastou Fatemi, Terence Verla (Duke University School of Medicine), Harrison Farber, Joseph S. Cheng MD MS (Vanderbilt University Medical Center), Carlos Antonio Bagley MD (Duke University Medical Center), Oren N. Gottfried MD (Duke University Mecical Center)

Introduction: Recently, there has been a shift toward critical analyses of quality and longitudinal assessment of the subjective and objective outcomes after lumbar spine surgery. Accordingly, the emergence and routine use of institutional registries have been vital to the longitudinal assessment of quality. However, prospectively obtaining longitudinal outcomes for patients 24 months after spine surgery remains a challenge. Here, we assess whether 12-month measures of treatment effectiveness accurately predict long-term outcomes (24 months).

Methods: A multi-institutional, prospective spine outcomes registry was utilized. Enrollment criteria included available demographic, surgical and clinical outcomes data. All patients had prospectively collected outcomes measures and minimum 2-year follow-up. Patient reported outcomes instruments (ODI, SF-36, VAS-BP/VAS-LP) were completed before surgery, then at 3, 6, 12, and 24 months after surgery. The Health Transition Index of SF-36 was used to determine the 1-year Minimum Clinical Important Difference (MCID) and logistic regression modeling was performed to determine whether achieving MCID at 1-year adequately predicted improvement and achievement of MCID at 24-months.

Results: The study included 1506 patients: 300 patients underwent ALIF, 606 patients underwent TLIF and 600 patients underwent XLIF. There was significant correlation between 12- and 24-month ODI ( $\mathrm{r}=0.82 ; \mathrm{p}<0.0001), \mathrm{SF}-36$ PCS $(\mathrm{r}=0.89 ; \mathrm{p}<0.0001)$, VAS-BP $(r=0.90 ; p<0.0001)$ and VAS-LP $(r=0.85 ; p<0.0001)$. For ALIF cohort, patients achieving MCID for ODI at 12-months were 13 fold $(\mathrm{p}<0.0001)$ more likely to achieve MCID at 24-months. Similarly, for TLIF and XLIF cohorts, patients achieving MCID for ODI at 12 -months were 13 -fold and 14 -fold $(\mathrm{p}<0.0001)$ more likely to achieve MCID at 24-months, respectively.

Conclusions: In a multi-institutional prospective study, patient centered measures of surgical effectiveness at 12 months adequately predict long-term (24-month) outcomes after lumbar spine surgery. Patients achieving MCID at 1-year were more likely to report meaningful and durable improvement by 24 months; the 12-months time-point is sufficient to identify effective versus non-effective patient care.

\section{The Effect of Parkinson's Disease on Patients Undergoing Lumbar Spine Surgery}

Branko Skovrlj MD (Mount Sinai School of Medicine), Javier Zabdi Guzman, Christopher A. Sarkiss BS (Mount Sinai Medical Center), Samuel K Cho, John M. Caridi MD (Mount Sinai Neurosurgery)

Introduction: Parkinson's disease (PD) is increasingly recognized as an important cause of spinal disorders requiring surgical correction. Spinal decompression and/or fusion can be complicated due to poor bone quality and severe muscular dysfunction in this patient population. The purpose of this study was to assess characteristics and outcomes of patients with PD undergoing lumbar spine surgery for degenerative conditions.

Methods: The National Inpatient Sample was examined from 2002 to 2011. Patients were included for study based on ICD-9-CM procedural codes for lumbar spine surgery and further substratified to degenerative diagnoses. Incidence and baseline patient characteristics were determined. Multivariable analysis including patient and hospital characteristics and major comorbidities was done to determine independent risk factors increasing incidence of lumbar fusion revision in PD patients.

Results: PD patients account for $0.9 \%$ of all degenerative lumbar procedures. PD patients are older $(72.3$ versus $65.2, \mathrm{p}<.0001)$ and are more likely to be male $(60.3 \%$ male, $\mathrm{p}<.0001)$. Mean length of stay (LOS) was increased in PD patients undergoing lumbar fusion (5.2 days versus 4.1 days, $\mathrm{p}<.0001)$ and lumbar fusion revision (6.6 days versus 4.8 days, $\mathrm{p}<.0001)$. Costs were $14.2 \%(\mathrm{p}<.0001)$ higher for lumbar fusion and $28.0 \%(\mathrm{p}<.0001)$ higher for lumbar fusion revision in PD patients when compared to those without PD. Multivariable analysis indicates that osteoporosis, fluid/electrolyte disorders, blood loss anemia, Medicare, Medicaid and, in particular, no insurance (OR 19.57, $\mathrm{p}<.0001$ ) are significant independent predictors of lumbar fusion revision in patients with PD.

Conclusions: PD patients undergoing lumbar surgery for degenerative conditions have increased LOS and costs when compared to patients without PD. Uninsured patients with PD, possibly due to advanced PD secondary to limited healthcare access, are nearly 20 times more likely to have a revision following the index surgery.

\section{Opioid-related Adverse Events with Lumbar Spine Surgery: Is the Risk Real?}

David W. Polly MD (University of Minnesota), Kevin L Ong PhD (Exponent), Scott Lovald, Edmund Lau, Mark Kurd MD, Kris Radcliff $M D$

Introduction: Although lumbar spine surgery has positive outcomes, as many as $22.4 \%$ of unplanned readmissions within 30 days post-surgery are related to pain (Pugely 2014). The most common pain management protocol is intravenous narcotics, but this may be associated with opioid-related adverse events (ORAE), such as respiratory complications and post-operative nausea and vomiting (PONV)(Kessler 2013). This study evaluated ORAE risk using a nationally representative sample of elderly lumbar spine surgery patients.

Methods: Using the 5\% Medicare physician/carrier claims data (2010-2012), lumbar laminectomy (LL) and fusion (LF) patients were identified. The 90-day postoperative risk of ORAEs was assessed, using ICD-9 diagnosis codes for respiratory complications, paralytic ileus, PONV, acute delirium, urinary retention, etc.

Results: From our analysis of 16,765 LF and 24,514 LL patients, respiratory complications (bradypnea, pulmonary insufficiency, asphyxia, and hypoxemia) were the most commonly diagnosed ORAE (LF: $12.3 \%$ and LL: $8.0 \%$ ). Urinary retention rates of $6.7 \%$ were diagnosed for both LF and LL patients. The rate of PONV was $4.1 \%$ and $3.1 \%$ following LF and LL, respectively, while the corresponding rates of acute delirium were $3.7 \%$ and $2.3 \%$, respectively. 
Paralytic ileus occurred in $2.5 \%$ and $1.5 \%$ of LF and LL patients, respectively. Older patients and patients with more comorbidities and of lower socioeconomic status had higher adjusted risks of respiratory complications and acute delirium. Risk factors for PONV were patients with more comorbidities and women, while risk factors for urinary retention were older age, more comorbidities, and men. Men had a higher risk of paralytic ileus.

Conclusions: Although opioids are effective and are the most common treatment protocol for acute postoperative pain after lumbar spine surgery, the reliance on opioid analgesics exposes patients to ORAEs. These complications not only adversely affect the patient but also increase healthcare costs. Future research should explore alternative approaches to limit opioid consumption and risk of ORAEs.

226. Role of Quantitative MRI Assessments in Predicting Surgical Outcome in Cervical Spondylotic Myelopathy Patients: Results from the Prospective, Multicenter AOSpine North American Study

Aria Nouri BA MD, Michael G. Fehlings MD PhD FRCSC FACS (Toronto Western Hospital), Lindsay Tetreault Bsc, Kristian Dalzell, Juan Jose Zamorano MD

Introduction: Cervical Spondylotic Myelopathy (CSM) is the commonest cause of spinal cord impairment in the elderly population worldwide. While MRI is the primary imaging modality for confirming the diagnosis, its role in predicting surgical outcome remains unclear. Using prospective and multicenter study data it is the purpose of the present study to assess the role of MRI in predicting the surgical outcome of patients treated for CSM.

Methods: Two hundred and seventy-eight patients with $=1$ clinical signs of myelopathy were enrolled and underwent decompression surgery. Complete baseline clinical and MRI data were available for 102 patients. MRI parameters measured included presence/ absence of signal change on $\mathrm{T} 1$ and $\mathrm{T} 2$, $\mathrm{T} 2$ signal quantitative factors, and anatomical measurements. A dichotomized postoperative mJOA score at 6-months was used to characterize patients with mild myelopathy $(=16)$ and those with substantial residual neurological impairment $(<16)$. Univariate analysis assessed the relationship between baseline parameters and outcome. Multivariate logistic regression was conducted following a conceptual division of variables into three groups: T1 signal analysis, T2 signal analysis and anatomical measurements.

Results: Baseline mJOA ( $\mathrm{p}<0.001$; OR=1.644, CI:1.326-2.037), maximum canal compromise (MCC) $(\mathrm{p}=0.0322 ; \mathrm{OR}=0.965$, CI:0.934-0.997), T2 hyperintensity ROI area $(\mathrm{p}=0.0422 ; \mathrm{OR}=0.67$, CI:0.456-0.986) and sagittal extent ( $\mathrm{p}=0.026$; OR=0.673, CI:0.4750.954 ) were significantly associated with outcome univariately. The final model was comprised of $\mathrm{T} 1$ hypointensity $(\mathrm{p}=0.029$; $\mathrm{OR}=0.242$; CI:0.068-0.866), MCC ( $\mathrm{p}=0.005$; OR=0.940; CI:0.90$0.982)$ and baseline mJOA ( $<<0.001 ; \mathrm{OR}=1.743$; CI:1.353-2.245), yielding an area under the receiver operating characteristic curve (AUC) of 0.845 .

Conclusions: Baseline mJOA is a strong predictor of postsurgical outcome in CSM at 6-months. However, a model inclusive of MCC and T1 hypointensity assessment provides superior predictive capacity. This suggests that MRI analysis has a distinct and significant role in predicting surgical outcome. It is therefore recommended that a thorough MRI analysis be conducted in all CSM patients considered for surgical treatment.
227. Anterior Cervical Discectomy and Fusion (ACDF): Comparison Between Zero Profile Implants and Anterior Cervical Plate and Spacer

Marjan Alimi MD (New York Presbyterian Hospital Weill Cornell Medical Center), Innocent Njoku BS (Weill Cornell Medical College), Christoph Hofstetter MD PhD, Kartik Kesavabhotla, Apostolos Tsiouris MD, John A. Boockvar MD (Weill Cornell Medical College), Roger Hartl MD (New York Hospital/Cornell Medical Center)

Introduction: Interposition grafts combined with anterior-plating currently remain the gold standard for anterior cervical discetomy and fusion. The use of anterior plates increases fusion rates, but may be associated with higher rates of postoperative dysphagia. The current study aimed to compare the clinical and radiological outcomes following anterior cervical discectomy and fusion (ACDF) using zero-profile anchored spacers versus standard interposition grafts with anterior-plating.

Methods: This was a retrospective case series. A total of 53 male and 51 female consecutive patients (164 total operated levels) who underwent ACDF between 2007 and 2011 were included. The mean clinical follow-up was $15.7 \pm 1.2$ (SEM) months for patient with zero-profile implants and $14.8 \pm 2.1$ months for patients with conventional ACDF with anterior plating. Patient demographics, operative details, clinical outcome, complications and radiographic imaging were reviewed. Dysphagia was determined using Bazaz criteria.

Results: Clinical outcome scores were similar between the two groups as measured by the modified JOA and Nurick scores. Zeroprofile constructs gave rise to significantly less prevertebral soft tissue swelling compared to constructs with anterior plates postoperatively $(15.74 \pm 0.52$ as compared to $20.48 \pm 0.85 \mathrm{~mm}, \mathrm{p}<0.001)$ and at the lastest follow-up $(10.88 \pm 0.39 \mathrm{~mm}$ vs. $13.72 \pm 0.67 \mathrm{~mm}, \mathrm{p}<$ $0.001)$. At latest follow-up, there was a significantly lower incidence of dysphagia associated with utilization of zero-profile constructs, as compared to anterior plate ( $1.5 \%$ vs. $20 \%, \mathrm{p}=0.001$, respectively).

Conclusions: Zero-profile implants lead to functional outcomes similar to standard anterior plate constructs. Avoiding use of anterior locking plate may decrease the risk of postoperative dysphagia.

\section{Occipitocervical Fixation: A Single Surgeon Experience} with 120 Patients

Eduardo Martinez del Campo Oviedo MD (Barrow Neurological Institute), Samuel Kalb MD (Barrow Neurological Institute), Jay D. Turner MD PhD (Barrow Neurological Institute), Luis PerezOrribo, Leonardo Rangel-Castilla MD (Barrow Neurological Institute), Hector Enrique Soriano-Baron MD (National Institute of Neurology and Neurosurgery), Nicholas Theodore M.D. F.A.C.S. (Barrow Neurosurgical Associates)

Introduction: Occipitocervical junction instability can lead to serious neurologic injury or death if left untreated. Open surgical fixation is often necessary in order to provide definitive stabilization.

Methods: The charts of all patients undergoing posterior internal fixation by the senior author were retrospectively reviewed. One hundred and twenty consecutive patients were identified for analysis. Patient demographics, occipitocervical junction pathology, surgical indications, clinical and radiographic outcomes are reported.

Results: The study population consisted of 64 males and 56 females, mean age of 39.9 years (range 7 months - 88 years). Trauma was the most common cause of instability, occurring in 56 patients $(46.7 \%)$. Ninety patients $(75 \%)$ were treated with screw/ rod constructs; wiring was used in 30 patients $(25 \%)$., The median number of fixated segments was $5(\mathrm{O}-\mathrm{C} 4)$. Structural bone grafts were implanted in most patients $(83.3 \%)$. Pre-operative neurological deficits were present in 83 patients $(69.2 \%)$. Overall neurological 


\section{Meeting Abstracts: AANS/CNS Section on Disorders of the Spine and Peripheral Nerves}

improvement was $90.9 \%$. Mean follow-up was 16.1 months (range 4-107 months). There was radiographic evidence of fusion in all patients $(100 \%)$. One patients suffered neurological deterioration and none died during the first year after surgery. The overall complication rate was $10.8 \%$, including 3 patients with vertebral artery injuries and 2 patients with hardware failures which required surgical correction.

Conclusions: OC fixation is a durable treatment option with acceptable morbidity for patients with OC instability.

\section{Comparison Between Hinged and Contoured Rods for Occipitocervical Arthrodesis}

Kingsley O. Abode-Iyamah MD (University of Iowa Hospitals and Clinic), Brian J. Dlouhy MD (St. Louis Children's Hospital), Patrick W. Hitchon MD (University Of Iowa Hospitals), Nader S. Dahdaleh MD (Northwestern University)

Introduction: Occipital plates and upper cervical spine screws are the most rigid constructs used in occipitocervical fusions. Rods are contoured to accommodate the craniovertebral junction angle. With acute bends the chances of biomechanical failure with repetitive cycle loading increases. Hinged rod systems were developed to obviate the need for rod bending and hence may improve fatigue failure. This is the first clinical study comparing hinged and contoured rod systems during occipitocervical arthrodesis.

Methods: 39 consecutive patients who underwent occipitocervical fusions with at least 6 months follow up from two tertiary care centers were retrospectively reviewed. Hinged rod systems were used in 19 patients while 20 patients received contoured rods. Basic demographics, indications, the use of $\mathrm{C} 1$ and $\mathrm{C} 2$ screws, bone graft, length of stay, estimated blood loss, surgical time, preoperative and postoperative occipitocervical angles, Frankel, and Nurick scores were compared. Flexion and extension cervical spine xrays during the last follow up was used to assess fusions.

Results: There was no difference in the mean age, gender distribution, length of constructs, and use of bone allograft between both groups. $\mathrm{C} 1$ screws were used in two patients receiving contoured rods and in none receiving hinged rods. Follow up was 12.2 and 12.6 months for hinged and contoured rod groups respectively. Preoperative, direct post-operative and last visit occipitocervical angles were 29,27 , and 26 degrees respectively for the contoured rod group and 26, 23, 23 degrees for the hinged rod group with no difference within the groups and between them. Similarly, EBL, length of stay, preoperative and postoperative Frankel and Nurick scores were similar between both groups. None of the patients required revision surgery for pseudoarthrosis or rod fracture in both groups.

Conclusions: Hinged rod systems can be used as an alternative to contoured rods during occipitocervical arthrodesis with similar clinical and radiographic outcomes.

230. Rate of 30-day Postoperative Myocardial Infarction in Patients Undergoing Spine Surgery

Timothy Y. Wang BS (Duke University), Joel Martin MD (University of California, San Diego), Daniel Loriaux, Oren N. Gottfried MD (Duke University Medical Center)

Introduction: There is an increased emphasis to reduce complications and improve outcomes after spinal surgery. One of the more devastating postoperative complications of elective and emergent spinal surgery is myocardial infarction (M.I.) and the incidence and risk factors for its occurrence are not well described.

Methods: We evaluated all medical records and imaging of 1346 consecutive patients who underwent spinal surgery at Duke University from 2010 to 2012 for incidence of myocardial infarction within 30 days of surgery and documented all demographic, preoperative, perioperative, and postoperative variables. Associations between postoperative MI and individual risk factors in all patients were determined using logistic regression analysis. Patients were stratified into emergent and elective groups and a similar analysis was performed.

Results: Overall, 22 patients (1.6\%) had a MI in the 30 days following surgery, 14 patients $(1.2 \%)$ undergoing elective surgery and 8 patients $(4.2 \%)$ after emergent surgery $(\mathrm{p}=.047)$. Without stratifying by emergent or non-emergent etiology, multivariate logistic regression determined that age $>65$ years, atrial fibrillation, hypertension, prior MI, anticoagulant use, low albumin, length of stay $>7$ days, intraoperative transfusion, trauma etiology, baseline creatinine $>1 \mathrm{mg} / \mathrm{dL}$, and $>2$ levels of spinal fusion were independent predictors for postoperative MI, while degenerative etiology and single-level fusion were identified as negative predictors. When stratified by emergent surgery, we found age $>65$ years and baseline creatinine $>2 \mathrm{mg} / \mathrm{dL}$ to be independently associated with increased risk of postoperative MI. When stratified by elective surgery, we found that age $>65$, postoperative stay $>7$ days, intraoperative blood transfusion, baseline creatinine $>1 \mathrm{mg} / \mathrm{dL}$, and fusion of $>1$ level were associated with increased risk of MI. Interestingly, common factors including diabetes, heart failure, CAD, obesity, hypercholesterolemia, and smoking were not identified as independent risk factors.

Conclusions: The present study demonstrates a low incidence of MI after elective surgery with a higher incidence after emergent spine surgery and identifies patient factors predictive of postoperative MI. Identification of these factors impacts discussions of risk assessment and surgical decision making.

231. Defining the Prevalence of Unidentified Psychological Comorbidity in a Referral Population for Spinal Cord Stimulation

Mohammed F. Shamji MD PhD (Division of Neurosurgery, Toronto Western Hospital), Alina Shcharinsky $R N(E C) M N$ CNN $(C)$, Jessica Rodriguez, BS

Introduction: Neuropathic pain has the potential to affect various dimensions of patient health, including physical, psychological, and socioeconomic. What remains unknown is spectrum of psychological dysfunction that accompanies this pain phenotype and whether there is a difference based on the etiology of the pain among patients referred for spinal cord stimulation (SCS).

Methods: We prospectively assessed patients with neuropathic pain referred for spinal cord stimulation for comorbid psychopathology. Demographic data included age, gender, diagnosis, marital status, and educational level of achievement. Screening tools were applied for both neuropathic pain diagnosis and for the presence of significant depression or anxiety disorder. Descriptive statistics were used to define the prevalence of these disorders, compared as well by gender and diagnosis. Logistic regression was used to correlate pain intensity with severity of psychopathology.

Results: Nearly a quarter of patients evaluated were excluded because they did not reach criteria for neuropathic pain suitable for management by SCS. Among the remaining patients, $54 \%$ were women, the median age was 51 , and $35 \%$ and $42 \%$ admitted to routine smoking and alcohol use respectively. The most common diagnoses were CRPS (46\%) and FBSS (38\%). Depression was screened in $60 \%$ of patients, more common among women and CRPS patients. Anxiety was screened in $25 \%$ of patients, more common among men and FBSS patients. In logistic regression, pain intensity was correlated with the presence and severity of depression $(\mathrm{p}<0.02)$ but not anxiety disorder $(\mathrm{p}=0.43)$.

Conclusions: In this cohort of patients assessed for spinal cord stimulation, there was a high prevalence of mood disorder, regardless of whether patients previously held that diagnosis. 


\section{Meeting Abstracts: AANS/CNS Section on Disorders of the Spine and Peripheral Nerves}

Depression was highly prevalent and correlated with pain intensity. Management of neuropathic pain in a spinal cord stimulation practice may benefit from concurrent mood optimization.

232. The Risk of Acute Spinal Cord Injury After Minor Trauma in Patients with Preexisting Cervical Stenosis

Victor Chang MD (Henry Ford Hospital), Langston T. Holly MD (University of California Los Angeles)

Introduction: The actual risk of acute SCI following minor trauma in cervical stenosis patients is unknown. This study seeks to evaluate the risk of SCI after minor trauma in a cohort of prospectively followed patients with cervical stenosis.

Methods: This study is composed of 55 nonoperatively treated, cervical stenosis patients evaluated between 2009 and 2014. Clinical and radiographical analysis was performed. Each patient was asked specific standardized questions including 1) whether a previous physician recommended neck surgery, 2) whether a physician told them that they would become paralyzed following a traumatic event, 3) whether they had suffered a traumatic event during the follow-up period.

Results: The mean age was 65 years old, with a mean mJOA of 16.6. The mean canal diameter was $6.1 \mathrm{~mm}$, and 19 patients $(35 \%)$ had evidence of intramedullary T2 signal abnormality. Thirty-one patients $(56 \%)$ were previously recommended surgery. Twenty-six patients $(47 \%)$ were told that they would be paralyzed following a motor vehicle accident or fall unless surgery was performed. Ten patients (18\%) experienced a traumatic event during the follow-up, with none sustaining an SCI.

Conclusions: Asymptomatic and mildly symptomatic cervical stenosis patients are commonly recommended to undergo surgical intervention due to risk of paralysis following a traumatic event. SCI was not observed following minor trauma in our cohort of prospectively followed patients. It appears that occurrence of SCI in this patient population following minor trauma is likely smaller than many physicians surmise, yet will require future prospective study in a large cohort of patients.

233. Early Behavioral Voiding Patterns Predict Long-term Urinary Dysfunction and Potential Recovery After Experimental Cervical Spinal Cord Injury

Sean Mitchell Aubuchon (University of Florida), Xiaoping Luo, Tyler Bogus, Hung-Yuan Chen, Daniel J. Hoh MD (University of Florida)

Introduction: Spinal cord injury(SCI) can result in devastating neurologic deficits. Among these, impaired lower urinary tract function(LUT) ranks as a leading cause of morbidity. Surveyed SCI individuals target recovery of LUT function as a top priority for research. Clinically, LUT impairment demonstrates variable dysfunction with some showing partial recovery of spontaneous bladder emptying, albeit via adaptive, incomplete pathways. Little experimental research, however, has investigated mechanisms and patterns that correlate with long-term dysfunction versus recovery. Development of translational therapies for improving LUT function after SCI depends on identifying early predictors of chronic disease. Therefore, the purpose of this study is to examine longitudinal LUT function after experimental SCI to determine predictors of long-term dysfunction.

Methods: 10 Sprague-Dawley rats underwent mid-cervical contusion. Voiding behavior was assessed pre-injury and 4, 6, 8, 12 weeks post-SCI using chromatographic evaluation of volitional (normal) vs. non-volitional (abnormal) bladder emptying. Bladder cystometry and sphincter EMG were performed at 12 weeks postSCI to assess for dyssynergy and supraspinal-spinal neuronal con- nectivity. Bladder wall histology and elastin content were evaluated for functional changes related to chronic urinary retention.

Results: All animals demonstrated acute LUT dysfunction after cervical contusion. Non-volitional behavioral voiding at 4 weeks post-SCI correlated with persistent abnormal bladder contractions and sphincter EMG dyssynergy at 12 weeks, and with increased bladder muscle hypertrophy and elevated elastin. Conversely, spontaneous recovery of volitional behavioral voiding at 4 weeks correlated with normal bladder contractions and sphincter EMG synergy at 12 weeks, and more normal bladder muscle histology and elastin content.

Conclusions: LUT impairment demonstrates varying degrees of long-term dysfunction versus recovery after experimental SCI, consistent with heterogeneity seen after clinical SCI. Early behavioral voiding by 4 weeks post-SCI predicts likelihood of recovery. Development of translational therapies may consider evaluation of these early predictors in future investigational studies.

234. Cervical Spondylotic Myelopathy: Does Surgical Approach Influence Post-operative Sagittal Alignment and Outcomes?

Michael G. Fehlings MD PhD FRCSC FACS (Toronto Western Hospital), Vincent Challier, Christopher I. Shaffrey MD FACS (University of Virginia), Han Jo Kim MD, Paul M. Arnold MD (Department of Neurosurgery), Jens Chapman MD (University of Washington), Themistocles Protopsaltis MD, Virginie Lafage PhD (NYU Langone Medical Center), Shian Liu, Renaud Lafage, Frank Schwab MD, PhD, Eric M. Massicotte MD (Toronto Western Hospital), Tim Yoon, Christopher P. Ames MD (University of California San Francisco Neuro Surgery), Justin K Scheer BS (University of California, San Diego)

Introduction: The effect of surgical approach on outcomes for cervical spondylotic myelopathy (CSM) is controversial. Fehlings et al, showed that patients treated with anterior techniques tend to be younger, less impaired, and had more focal pathology. In this study we compared outcomes of an anterior approach group (AAG) versus a posterior approach group (PAG) with a focus on the effect of sagittal alignment.

Methods: Post-hoc analysis of a prospective, multicenter database of patients with CSM. 117 patients met inclusion criteria, were nonrandomized to an AAG ( $n=51)$ or PAG $(n=62)$, with post-operative static lateral radiographs, Nurick assessment, and HRQOLs at 6 months and/or 1 year. The AAG underwent anterior decompression and fusion, PAG either laminoplasty or laminectomy with fusion. Sagittal regional and focal parameters were compared by multivariate regression.

Results: At baseline, the groups showed significant age difference (AAG 51 yrs, PAG 62 yrs, $\mathrm{p}<0.001$ ), rheumatological comorbidity (AAG 3\%, PAG 19\%, p=0.011), and modified Japanese Orthopaedic Association (mJOA). The PAG had significantly more regional malalignment at baseline but there were no focal alignment differences between the two. Surgical features were significantly different for greater than 3 levels treated (AAG 27\%, PAG 97\%, $\mathrm{p}<0.001$ ) and blood loss (AAG $152 \mathrm{~mL}$, PAG $380 \mathrm{~mL}, \mathrm{p}<0.001$ ). After surgery, both AAG and PAG improved significantly in mJOA and Nurick grade, however there was no statistically significant difference in mJOA post-operatively. AAG had a decrease in sagittal vertical axis SVA while the PAG had a significant increase in SVA. There were no relevant post-operative focal alignment differences.

Conclusions: The PAG had older patients with more disability. Even when age, baseline mJOA, and regional parameters were controlled, the PAG cohort still correlated with worse CGH_C7 SVA and TS-CL. It is interesting to note the PAG had a lower mJOA than the AAG at baseline, they both improved and were not significantly different post-operatively. This suggests that both techniques relieve symptomatic disease, but patients in the posterior group may continue to progress in sagittal misalignment. 


\section{Meeting Abstracts: AANS/CNS Section on Disorders of the Spine and Peripheral Nerves}

\author{
235. Outcomes of Intact Thoracolumbar Burst Fractures: \\ Operative vs. Non-operative Treatment
}

Patrick W. Hitchon MD (University Of Iowa Hospitals), Nader S. Dahdaleh MD (Northwestern University), Christopher I. Shaffrey MD FACS (University of Virginia), Jennifer Noeller ARNP, Wenzhuan He MD MS, Toshio Moritani MD PhD (Uiowa Department of Radiology), Kingsley O. Abode-Iyamah MD (University of Iowa Hospitals and Clinic)

Introduction: The authors compare the clinical and radiographic outcome of intact thoracolumbar burst fractures treated operatively and non-operatively.

Methods: Since 2001, 75 intact patients with burst fractures of the thoracolumbar spine were seen at our institution and followed prospectively. Forty-eight patients, 25 women and 23 men, with ages $49 \pm 19$, were treated non-operatively. Twenty-seven patients, 17 men and 10 women, with ages $47 \pm 17$ years, underwent surgery.

Results: On admission, the residual canal $(51 \pm 13 \%)$ in the operative cohort was significantly smaller than the non-operative $(62 \pm 12 \%, \mathrm{p}=0.0005)$. Also, the PLC was more frequently disrupted in the operative cohort $(9 / 18)$ compared to the non-operative $(3 / 45$, $\mathrm{p}=0.002)$. Length of hospitalization was longer in the surgical group $(12+/-6$ vs. $7+/-6$ days, $\mathrm{p}=0.001)$. At follow-up, the final local kyphosis was significantly smaller in the operative $\left(2^{\circ}+/-10\right)$ than in the non-operative cohort $\left(14^{\circ}+/-8, \mathrm{p}=0.0000\right)$. Hospital charges were significantly higher for the operative cohort $(\mathrm{p}<0.0000)$, and at follow-up, there was no difference between groups in the VAS and Oswestry scores.

Conclusions: Residual canal and the post ligament status are significant parameters that can be used to predict allocation to the operative or non-operative group. If the PLC is intact, surgery is less likely (Odds ratio of surgery/no surgery $=0.096,95 \%$ CI $[0.022,0.416], \mathrm{p}=0.002)$. Similarly, the larger the residual canal, the less likely surgery will be needed (Odds ratio of surgery/no surgery $=0.947$ (95\% CI [0.911, 0.984]; $\mathrm{p}=0.005)$. Both operative and non-operative treatment modalities are associated with comparable favorable outcomes, and radiographic parameters should be used in selecting the more appropriate.

236. Bracing After Surgical Instrumentation of Thoracolumbar Fractures: A Systematic Review of Practices, Evidence and Indications

Jesse Skoch MD, Carmine Zoccali MD, Nikolay L. Martirosyan $M D$ (University of Arizona), Orel Zaninovich BS, Christina $M$. Walter MS, Philip Maykowski BA, Ali A. Baaj MD (University of Arizona)

Introduction: The role of spinal orthotic braces after surgical stabilization, is not clearly defined. We systematically reviewed the published literature to determine patterns of practices, indications and current evidence for the use of braces after thoracolumbar fracture stabilization.

Methods: An electronic literature search was performed in the National Library of Medicine for publications from 1990 to 2014. Search criteria included keywords related to spinal trauma, surgery, and bracing. The review analyzed specific surgical criteria: anterior, posterior, or circumferential, open or minimally invasive, whether a fusion was performed, the segmental length of instrumentation, and examined the correlation with the use of postoperative bracing. Differences between wearing vs. not-wearing a post-operative brace were examined with regard to loss of correction, residual pain, the ability to maintain previous work activity, pseudoarthrosis, and the percentage of reported complications. A chi-square test for independence was used to determine relationships.

Results: Our search yielded 78 pertinent studies. Postoperative bracing (POB) was adopted in 60 out 78 studies for a median wear- ing time of 3 months (Figure 1). Postoperative bracing was adopted in $81.3 \%$ of the posterior surgeries, $76.5 \%$ of anterior, and $66.7 \%$ of circumferential surgeries. In posterior operations, POB was adopted in $82.1 \%$ of cases where fusion was performed and in $76.5 \%$ where fusion was not performed. No differences in term of residual pain, return to work, pseudoarthrosis rate, or general complications were found between the POB and non-POB groups (Table 1\&2).

Conclusions: Most surgeons utilize braces for three months after surgical thoracolumbar fracture stabilization. Given the lack of clinical or biomechanical evidence for this, and the additional costs and potential discomfort to patients, further studies in this area are warranted to determine when and if POB for surgically stabilized thoracolumbar fractures is indicated.

237. Safety of L4-5 Lateral Interbody Fusion via Minimally Invasive Lateral Transpsoas Approach: The Long Term Clinical Experience

Gisela Murray MD, Joshua Marshall Beckman MD (University of South Florida), Konrad Bach MD (University of South Florida), Juan S. Uribe MD (University of South Florida - Division of Neurosurgery)

Introduction: Minimally invasive lateral transpsoas approach at L4-L5 has been associated with higher morbidity risk than other lumbar levels, due to the proximity of lumbar plexus and vascular structures. However, if the anatomical safe zone corridor is followed, this level can be equally safe. This study examines the long-term clinical and radiological outcomes of L4-5 lateral interbody fusion (LIF).

Methods: Retrospective analysis was performed for patients with L4-5 LIF exclusively. Records were reviewed for demographic data, surgery characteristics and development of complications. Outcomes measures used to evaluate long-term clinical success were visual analog scale (VAS) score, Oswestry Disability Index (ODI), SF-12, and SF-36. Radiographic parameters measured were segmental Cobb angle, lumbar lordosis, and foraminal and disc height.

Results: Forty-nine patients underwent L4-5 LIF from 20102014. The mean age was 60 years. Thirty-eight patients $(78 \%)$ were female. Most common diagnosis was spondilolithesis (80\%). EBL was $35 \mathrm{ml}$. The mean follow up was 17 months. Fusion rate was $96 \%$. Iliopsoas weakness developed in 5 patients; all but 2 resolved at follow up. One patient suffered a contralateral femoral nerve injury after developing a contralateral large psoas hematoma due to segmental artery injury. Four patients experienced anterior thigh numbness. No infections or visceral injury were seen. Four patients required revision surgery: two for posterior decompression at index level, one for adjacent level failure and one for evacuation of the psoas hematoma. Postoperatively, ODI, VAS and SF physical health scores decreased significantly. Radiographic parameters demonstrated an increase in the disc and foramina height.

Conclusions: Minimally invasive lateral transpsoas interbody fusion has demonstrated to be a safe procedure for L4-5 pathology. This technique successfully reconstructs anterior column support, with restoration of disc and foraminal height. In spite of the distinct anatomy, the L4-5 LIF is associated with minimal complications, low blood loss and favorable clinical outcome.

238. Can Triggered Electromyography Monitoring Throughout Retraction Predict Postoperative Symptomatic Neuropraxia after XLIF? Results from a Prospective Multicenter Trial

Juan S. Uribe MD (University of South Florida - Division of Neurosurgery), Jim A. Youssef MD, Robert E. Isaacs MD (Duke University Medical Center), SOLAS Degenerative Study Group

Introduction: This multicenter study aims to evaluate the utility of triggered electromyography (t-EMG) recorded throughout psoas 


\section{Meeting Abstracts: AANS/CNS Section on Disorders of the Spine and Peripheral Nerves}

retraction during lateral transpsoas interbody fusion to predict postoperative changes in motor function.

Methods: Three hundred twenty three (323) patients undergoing L4-5 minimally invasive lateral interbody fusion from 21 sites were enrolled. Intraoperative data collection included initial t-EMG thresholds of the posterior blade from the stimulated retractor and subsequent t-EMG threshold values collected every five minutes throughout retraction. Additional data collection included dimensions/duration of retraction as well as pre-and postoperative lowerextremity neurologic exams.

Results: Prior to expanding the retractor, the lowest t-EMG threshold was identified posterior to the retractor in $94 \%$ of cases. Postoperatively, 13 (4.5\%) patients had a new motor weakness that was consistent with symptomatic neuropraxia (SN) of lumbar plexus nerves on the approach side. There were no significant differences between patients with or without a corresponding postoperative $\mathrm{SN}$ with respect to initial posterior blade reading $(\mathrm{p}=0.600)$, or retraction dimensions $(\mathrm{p}>0.05)$. Retraction time was significantly longer in those patients with SN vs. those without ( $p=0.031)$. Stepwise logistic regression showed a significant positive relationship between presence of new postoperative $\mathrm{SN}$ and total retraction time $(\mathrm{p}<0.001)$, as well as change in t-EMG thresholds over time $(\mathrm{p}<0.001)$.

Conclusions: Prolonged retraction time and increasing t-EMG thresholds throughout retraction are predictors of declining nerve integrity. In addition to a careful approach with minimal muscle retraction and consistent lumbar plexus directional retraction using dynamically triggered discrete-threshold t-EMG, the incidence of postoperative motor neuropraxia may be reduced by limiting retraction time and utilizing t-EMG consistently throughout retraction.

\section{Hip Flexion Weakness After XLIF}

Juan S. Uribe MD (University of South Florida - Division of Neurosurgery), Jim A. Youssef MD, Robert E. Isaacs MD (Duke University Medical Center), SOLAS Degenerative Study Group

Introduction: Access to the disc space in the lateral approach to the lumbar spine is gained by traversing the lumbar plexus and psoas muscle. In some patients this muscle retraction results in pain that manifests as hip flexion weakness (HFW). The purpose of this analysis is to evaluate the relationship between intraoperative metrics and the presence of post-operative HFW.

Methods: As part of a study on EMG monitoring throughout the XLIF procedure, patients from 21 sites who underwent XLIF at L4-5 with or without treatment at additional levels were enrolled in a prospective, nonrandomized, multi-center, institutional review board (IRB)-approved, clinical study. Data collection included demographics, pre-and postoperative 0-5 motor function and intraoperative collection of total number of interbody levels fused, total procedure time, estimated blood loss, and use of bone morphogenetic protein (BMP). Results from patients with postoperative hip flexion-weakness were compared to those without.

Results: Three hundred twenty three (323) patients were enrolled. Postoperatively 167 had no decrease in motor or sensory function, 91 had HFW with or without additional motor/sensory decreases in function. The remaining 65 patients were removed from analysis due to either missing data or the presence of postoperative decreases in motor/sensory function without HFW. Women vs. men were more likely to develop HFW (45\% of females vs. $18 \%$ of males, $\mathrm{p}<0.001)$, as were smokers vs non-smokers $(51 \%$ of smokers vs. $33 \%$ of non-smokers). Factors that were not significantly different between patients with and without HFW were body mass index $(\mathrm{p}=0.301)$, age $(\mathrm{p}=0.871)$, preoperative diagnosis of scoliosis $(\mathrm{p}=0.0711)$, number of levels fused $(\mathrm{p}=0.095)$, intraoperative use of BMP $(\mathrm{p}=0.221)$, total procedure time $(\mathrm{p}=0.292)$, and estimated blood loss $(\mathrm{p}=1.00)$.

Conclusions: In this population, HFW was greater in women and patients who smoked. On average, patients who developed postoperative HFW were treated at more levels, though this measure was not significantly different.

240. The Effects of Cage Size on Motor and Sensory Nerve Function During the XLIF® Procedure: Preliminary Results from a Prospective, Multi-center Study

Robert E. Isaacs MD (Duke University Medical Center), Jim A. Youssef MD, Juan S. Uribe MD (University of South Florida Division of Neurosurgery), SOLAS Degenerative Study Group

Introduction: The availability of increasingly wide and increasingly tall PEEK implants are available for lateral lumbar interbody fusion. While a larger cage size has the advantage of indirect decompression through elevated disc height as well as added stability, insertion of a larger cage may require larger retraction of the psoas muscle and consequently a greater risk of injury to the surrounding tissue. The purpose of this study is to evaluate the effect of L4-5 cage size on postoperative changes in nerve function.

Methods: Patients from 21 sites who were undergoing XLIF at L4-5 were enrolled in a prospective, nonrandomized, multi-center, institutional review board (IRB)-approved, clinical study to evaluate the utility of triggered EMG throughout lateral retraction. As part of that research effort Intraoperative data collection included the interbody cage sizes and retraction size in the anterior/posterior and cranial/caudal directions. Pre-and postoperative 0-5 lower-extremity motor function were collected to identify new postoperative motor deficits.

Results: Three hundred eighteen (323) patients were enrolled. Postoperatively, 13 (4.0\%) patients had symptomatic motor neuropraxia on the approach side. The most prevalent cage dimensions were $22 \mathrm{~mm}$ width $(60.0 \%), 55 \mathrm{~mm}$ length $(45.3 \%)$, and $10 \mathrm{~mm}$ height $(54.2 \%)$. Retractor clicks in the anterior-posterior direction were positively correlated with cage width $(\mathrm{p}<0.001)$, and clicks in the cranial-caudal direction were positively correlated with cage length $(p=0.005)$ and height $(p=0.007)$. Incidence of nerve injury, psoas weakness, post-op motor weakness distal to the psoas, and post-op sensory reduction were not significantly different across various cage dimensions.

Conclusions: The results of this study provide evidence that longer and wider interbody cages are predictors of larger retraction in the cranial-caudal direction, but not risk for increasing incidence of decreased motor or sensory function.

241. Morbidity of Repeat Surgery for Recurrent Spinal Metastases Following Combined Separation Surgery and Stereotactic Radiosurgery

Vijay Yanamadala MD (Massachusetts General Hospital), Joseph Schwab MD, Kristina Shultz, Kevin MD Oh, John H. Shin MD (Massachusetts General Hospital)

Introduction: Complications associated with surgery after conventional radiotherapy are known and include delayed wound healing and poor functional outcomes. Stereotactic radiosurgery (SRS) is an effective post-operative adjuvant for local tumor control. Limited data is available regarding the morbidity of additional surgery following SRS and separation surgery.

Methods: All surgeries for spinal metastases treated with postoperative SRS at a single institution between 2012 and 2014 were retrospectively reviewed. Patients who were treated with SRS alone were excluded.

Results: Thirty seven patients were treated with SRS (18Gy, 1 fraction) after separation surgery. Five patients (ages 54-77; 4 male, 1 female; 3 thoracic, 2 lumbar) required repeat surgery for local tumor recurrence $(n=4)$ or pathological fracture $(n=1)$. Four 


\section{Meeting Abstracts: AANS/CNS Section on Disorders of the Spine and Peripheral Nerves}

patients had complications related to surgery (80\%). Histologies included breast, lung, renal cell, hepatocellular, and esophageal. Three patients had radiotherapy prior to SRS (13,39,48 months). Four patients developed local progression at the treated site (6-23 months from the time of SRS, mean 12.5 months) and one developed symptomatic fracture 12 months after SRS. The 4 patients with local failure had progression of systemic disease at the time of repeat surgery. With a mean post-reoperation follow-up of 5.8 months, 3 patients passed away (survival range 3 months-1 year). One patient is living with preserved function (KPS 80, ECOG 1) and the other developed recurrent cord compression 1 year after repeat surgery (KPS 50, ECOG 3). Three patients had wound defects with a mean of 2.6 months until full closure. Of these, 1 required 3 further surgeries. One patient had an epidural hematoma requiring evacuation.

Conclusions: This is the first series reporting morbidity of repeat surgery following surgery and SRS. Though there may be clinical benefit to repeat surgery, the associated complications are high. Though this is a small sample size, further reporting will help identify high risk patients.

242. Posterior Thoracic Corpectomies with Cage Reconstruction for Metastatic Spinal Tumors: Comparing the Mini-Open Approach to the Open Approach

Darryl Lau MD (University of California, San Francisco), Dean Chou M.D. (University of California San Francisco)

Introduction: There has been a shift of practice to utilize posterior-only approaches to performing thoracolumbar corpectomies for spinal metastasis, but most are still done through an open approach. This study compares outcomes of patients who undergo mini-open vs. traditional open transpedicular corpectomy for thoracic spinal metastasis.

Methods: A consecutive cohort of adult patients from 2006 to 2013 who underwent transpedicular corpectomy in the thoracic spine for spinal metastasis was retrospectively identified. The miniopen transpedicular corpectomy was performed with a midline facial incision only over the level of corpectomy of interest and percutaneous instrumentation. Bivariate statistics were employed to compare perioperative and follow-up outcomes between open surgery and mini-open surgery.

Results: A total of 49 patients were included: 21 patients had mini-open surgery, and 28 patients had open surgery. Mean age was 57.9 years, and $59.2 \%$ were male. The two groups were well matched. There were no significant difference in operative time between the open and mini-open groups (413.6 minutes vs. 452.4 minutes, $\mathrm{p}=0.329$ ). Compared to the open group, the mini-open group had significantly less blood loss $(1697.3 \mathrm{cc}$ vs. $917.7 \mathrm{cc}$, $\mathrm{p}=0.019)$ and significantly shorter length of hospital stay (11.4 days vs. 7.4 days, $\mathrm{p}=0.001)$. There was a trend towards a lower perioperative complication rate in the mini-open group (9.5\%) compared to the open group $(21.4 \%)$, but this was not significant $(\mathrm{p}=0.265)$. At follow-up, there were no significant differences in ASIA score $(\mathrm{p}=0.342)$, complication rate after 30 -days $(\mathrm{p}=0.999)$, and surgical revision rate $(\mathrm{p}=0.803)$. The open approach had a higher overall infection rate of $17.9 \%$ compared to the mini-open approach $(9.5 \%)$, but this was not statistically significant $(\mathrm{p}=0.409)$.

Conclusions: The mini-open transpedicular corpectomy is a safe and feasible procedure and offers less blood loss and shorter length of hospital stay. The mini-open corpectomy also trended towards lower infection rate and lower complication rate.
243. Estimating Incremental Cost-Effectiveness of Adult Spinal Deformity Surgery Using the SRS-Scwhab Classification System

Chessie Robinson MA (4708 Alliance Blvd), Richard A. Hostin MD (Southwest Scoliosis Institute / Baylor Scoliosis Center), Michael F. O'Brien MD, Christopher P. Ames MD (University of California San Francisco Neuro Surgery), Frank Schwab MD, PhD, Justin S. Smith MD PhD (University of Virginia Health System), Virginie Lafage PhD (NYU Langone Medical Center), Munish Gupta MD (University of California - Davis; Ortho Surgery), Eric Klineberg MD (University of California - Davis), Robert Hart MD (Ohsu Spine Center), Ian McCarthy PhD (4708 Alliance Blvd.)

Introduction: We evaluate the incremental cost-effectiveness of adult spinal deformity surgery relative to nonsurgical treatment using the SRS-Schwab sagittal modifier classification.

Methods: We conduct a retrospective analysis of consecutive surgical and nonsurgical ASD patients. Surgical data was from a single center observational dataset while nonsurgical data was from a prospective multi-center database. Hospital costs were collected from administrative data and QALYs were calculated from the SF-6D, each discounted at 3.5\% annually. We analyzed the incremental cost-effectiveness ratios (ICERs) based on sagittal modifier at baseline. The 3 modifier types are mild $(\mathrm{SVA}<4 \mathrm{~cm}$, $\mathrm{PT}<20^{\circ}$, PI-LL within $10^{\circ}$ ), moderate (at least 1 of SVA $4-9.5 \mathrm{~cm}$, PT $20^{\circ}-30^{\circ}$; PI-LL $10^{\circ}-20^{\circ}$, and no severe modifiers); and severe (at least 1 of SVA $>9.5 \mathrm{~cm}, \mathrm{PT}>30^{\circ}, \mathrm{PI}-\mathrm{LL}>20^{\circ}$ ). The difference in QALYs between the surgical and nonsurgical cohorts at 2-years was projected through 5-year follow-up. 95\% confidence intervals were calculated using nonparametric bootstrap methods.

Results: Two-year follow-up was available for 278 patients (168 surgical, 110 non-surgical), predominantly female ( $\mathrm{n}=245,88 \%)$ with average age of $52(18-81)$. Total surgical costs averaged $\$ 121,857$, including readmissions. The 2 -year ICER was least costeffective for patients with severe sagittal modifiers at $\$ 424,978$ and most cost-effective for patients with mild modifiers at $\$ 206,730$. Projected through 5 years, the ICER among patients with severe sagittal modifiers was $\$ 151,689$, reducing to $\$ 73,790$ for patients with mild modifiers.

Conclusions: Based on the World Health Organization's suggested upper threshold for cost-effectiveness (3 times GDP, or $\$ 150,000)$, ASD surgery appears cost-effective after a 5-year period for mild and moderate sagittal modifier types. While average 5-year ICERs for severe modifier types are above this threshold, this value is included within the confidence interval, indicating the 5-year ICER is not statistically significantly higher than the threshold value. The ICER is expected to further decrease over extended follow-up.

244. Surgical Outcomes for Minimally Invasive Versus Open Transforaminal Lumbar Interbody Fusion (TLIF): An Updated Systematic Review and Meta-Analysis

Aaron J. Clark MD PhD (Semmes-Murphey Neurologic \& Spine Institute), Nickalus Khan MD (University of Tennessee Health Science Center), Siang Liao Lee MD, Garrett T. Venable $B S$, Nicholas Rossi MD (University of Tennessee Health Science Center), Kevin T. Foley MD FACS FAANS (Semmes-Murphey Neurologic \& Spine Institute)

Introduction: Minimally invasive TLIF(MI-TLIF) has been increasing in prevalence as compared to open TLIF(O-TLIF) procedures. MI-TLIF is an evolving technique with conflicting reports in the literature regarding outcomes. The goal of this study was to investigate the impact of MI-TLIF compared to O-TLIF on early and late surgical outcomes using the VAS and ODI scales. Secondary end points included blood loss, operative time, radiation 
exposure, length of stay, fusion rates, and surgical complications for the two procedures.

Methods: A systematic literature search using AMSTAR and PRISMA guidelines identified 987 articles that related to TLIF. Of these, 30 met inclusion criteria and directly compared MI and O-TLIF. A random effects meta-analysis was performed using both pooled and subset analysis based on study type.

Results: The 30 identified studies were assigned an average grade of $4.72 \pm 0.49$ stars using the Newcastle-Ottawa Scale. They comprised one randomized controlled trial, 12 prospective studies, and 17 retrospective studies. Our meta-analysis demonstrated that MI-TLIF was associated with reduced blood $\operatorname{loss}(\mathrm{p}<0.00001)$, length of stay $(\mathrm{p}<0.00001)$, and surgical complications $(\mathrm{p}=0.0008)$ as compared to O-TLIF but increased radiation exposure $(\mathrm{p}<0.00001)$. No differences were found in fusion rate $(\mathrm{p}=0.61)$ and operative time $(\mathrm{p}=0.34)$. An increased improvement in late VAS scores was demonstrated for MI-TLIF( $p<0.001)$ but no differences were found in improvements in early VAS, early ODI, and late ODI compared to O-TLIF.

Conclusions: MI-TLIF is associated with reduced blood loss, length of stay, complication rates, and increased radiation exposure as compared to O-TLIF. Differences in fusion rates, operative time, and early clinical improvement are insignificant. MI-TLIF may be associated with an increased improvement in late VAS scores compared to O-TLIF but yields similar improvements in ODI. More research is needed in the form of large, multi-institutional, prospective randomized controlled trials to compare these procedures.

245. Computer-assisted Navigation for Real Time Planning of Pedicle Subtraction Osteotomy for Cervicothoracic Deformity Correction

Vijay Yanamadala MD (Massachusetts General Hospital), Thomas D. Cha MD, John H. Shin MD (Massachusetts General Hospital)

Introduction: Pedicle subtraction osteotomy (PSO) is a powerful strategy for correction of cervicothoracic kyphotic deformity. Significant challenges to performing PSO in this region relates to the difficulty of visualizing the depth and extent of osteotomy due to the position of the head relative to the shoulders when prone. Visualization with fluoroscopy is often difficult and either breach through the ventral cortex or inadequate release potentially limits the corrective benefit of PSO. We describe the use of CT-guided real-time navigation to increase the accuracy of planning and performing PSO.

Methods: In each case of PSO, an intraoperative O-arm CT scan is performed with images relayed to the navigation system. After instrumentation is placed and the $\mathrm{C} 7$ pedicles identified, a navigation probe is used frequently to check the depth and trajectory of pedicle drilling and osteotomy, verifying the working angle. Axial and sagittal reconstructions during navigation provide instant feedback. Screenshots of the planned trajectories are saved and referenced during the PSO.

Results: Four patients (age 50-67, mean 56; 3 female, 1 male), three with severe cervical kyphotic deformity (mean chin-brownto-vertical-angle $25^{\circ}$; mean C2-7 Cobb angle $49^{\circ}$; mean T1 slope $17^{\circ}$ ) with progressive myelopathy (mJOA score $<13$ ) and one with severe thoracic kyphotic deformity, are included. Cervical PSO was performed at $\mathrm{C} 7$ in all cases. Thoracic PSO was performed at T5. There were no neurologic complications. Post-operative standing scoliosis xrays demonstrate preserved correction, mean CBVA $4^{\circ}$; C2-7 angle $17^{\circ}$; T1 slope $40^{\circ}$ with mean PSO correction of $24^{\circ}$ at mean 6 months follow-up. CT performed post-op confirms the accuracy of navigation in all cases.

Conclusions: This is the first series describing the use of CT-based navigation to aid in the planning accuracy of PSO for correction of cervicothoracic deformity. Integrating navigation into the workflow for these cases potentially improves the safety and outcomes of these cases.

\section{C-Arm Positioning is a Significant Source of Unnecessary} Radiation Exposure

Elizabeth W Reiser BA (Duke University Medical Center), Sarah Byrd NP-C, MSN (Duke University Hospital), Cary Idler $M D$, Robert E. Isaacs MD (Duke University Medical Center), Kris Radcliff MD, Frank M. Phillips MD (Midwest Orthopaedics at Rush)

Introduction: It is well-known that radiation exposure during minimally invasive spine procedures can be substantial, but less interest has focused on radiation exposure occurring in the OR before the procedure begins. Often, many images are taken prior to the procedure to determine proper entry points and angles.

Methods: From an IRB-approved database of over 1100 procedures, the minimally invasive spine cases for which set-up radiation was recorded were extracted. The amount of total radiation, set-up radiation, and procedure type were evaluated. Statistics were generated using a chi squared analysis.

Results: Setup and total radiation data were collected for 270 spine surgeries performed by four surgeons at two locations. 30 were thoracic and 240 were thoracolumbar or lumbar. There were 78 anterior cases and 192 posterior cases. Overall, average radiation recorded during the entire procedure (set-up, positioning, and surgery) was $8.04 \mathrm{rad}$, of which the average set-up amount was 1.90 $\operatorname{rad}(28 \%$, std $2.97 \mathrm{rad})$. For cases involving only the thoracic spine, this percentage was $52 \%$ with averages of $3.07 \mathrm{rad}$ for setup and $7.08 \mathrm{rad}$ total. The lumbar and thoracolumbar cases averaged $25 \%$ for setup/total, $1.76 \mathrm{rad}$ average setup for 8.16 total rad. Not only do thoracic cases get over twice the setup/total percentage compared to thoracolumbar or lumbar only cases, but statistically they also get more overall radiation during setup $(\mathrm{p}=0.02)$. Nonetheless, the amount of positioning radiation during all procedures was over a quarter of the procedure total.

Conclusions: Radiation exposure during C-arm positioning can be substantial, especially in thoracic cases where $90 \%$ of US spine physicians use fluoroscopy to localize in that region. Everyone in the OR should recognize the high percentage of radiation that occurs during $\mathrm{C}$-arm positioning, and merit should be given to technologies that can limit unnecessary radiation exposure during this portion of the procedure.

247. Comparative Biomechanics of Bryan and Pro-C Cervical Artificial Discs in a Caprine Model - Does Load Response Effect the Development of Heterotropic Ossificans?

Jamie Baisden MD (Medical College of Wisconsin), Gerald Eckardt MD (Medical College of Wisconsin), Joe Cochran MD (University of Texas at Houston Program), Frank A. Pintar $P h D$ (Medical College of Wisconsin), Narayan Yoganandan PhD (Medical College of Wisconsin)

Introduction: This caprine study is a biomechanical comparison of 2 FDA-approved artificial discs that are available for the treatment of cervical disc disease: Bryan Disc(BD), an unconstrained model;and ProDisc-C(PD), a semiconstrained model. CAD's were compared to ACDF.

Methods: ACDF $(n=4), \operatorname{PDC}(n=5)$, and BD $(n=6)$ were inserted at C3-4. Animals monitored for $6 \mathrm{mo}$ and $\mathrm{C}$-spines harvested. CTs were analyzed(3 neurosurgeons). Physiologic flexion-extension, axial rotation, lateral bending test was performed. Sub-failure axial compression and combined compression-flexion loads were applied in addition to pure moment loads. Final test was under axial loading to failure. Compression and compression-flexion responses were 


\section{Meeting Abstracts: AANS/CNS Section on Disorders of the Spine and Peripheral Nerves}

obtained. Response corridors were derived for ACDF, PD- and BD-type CADs, and intact animals.

Results: BD specimens observed a higher incidence of HO. PD specimens had minimal HO. Patterns of moment-rotation, forcedeflection and stiffness responses were similar for all models. BD flexion loading response was closer to the intact spine: BD, PD, ACDF: $21 \%, 45 \%, 70 \%$.

Conclusions: $\mathrm{CAD}$ and $\mathrm{ACDF}$ withstood higher forces than intact,(fig 1). BD not showing $\mathrm{HO}$ had biomechanical properties similar to intact (fig 2). BD was closer to intact than PD under flexion loading. PD demonstrated stiffer response than BD but with less ossification. The proposed benefit of CDA is in motion preservation and potentially to decreased adjacent segment disease. $\mathrm{HO}$ potentially negates the attempt at motion preservation and may lead to fusion. It is uncertain if abnormal load response, hypermobility of the motion segment, or potential inflammatory response to the implant may be the mechanism for development of HO. Our caprine model shows similar responses to human studies and suggests abnormal load response as a more probable mechanism for $\mathrm{HO}$ development in CDA. This load response may be important in the military population who are favoring CAD's over ACDF in efforts to maintain ROM.

248. Do MIS Deformity Interventions Result in Similar Reductions of Disability When Compared with Traditional Open Spinal Deformity Correction at 1 and 2 Years? A Propensity Matched Cohort Analysis

Kai-Ming G. Fu MD PhD (Weill Cornell Medical College), Adam S. Kanter MD (University of Pittsburgh Medical Center Presbyterian), Gregory Mundis MD (San Diego Center for Spinal Disorders), Paul Park MD (University Of Michigan, Department of Neurosurgery), Christopher I. Shaffrey MD FACS (University of Virginia), Frank LaMarca MD, Michael Y. Wang MD FACS (University of Miami), Juan S. Uribe MD (University of South Florida - Division of Neurosurgery), Richard G. Fessler MD, Praveen V. Mummaneni MD (University of California San Francisco Spine Center)

Introduction: Corrective surgery for adult spinal deformity (ADS) provides long term benefits but often at the cost of significant perioperative morbidity. The use of minimally invasive surgery (MIS) for correction of ADS has been theorized to lower the perioperative morbidity when compared to traditional open surgical approaches. However, there is concern that patients treated with MIS techniques will not achieve the same level of clinical improvement as those treated with traditional open surgery approaches. This study compared patients treated with MIS (MIS lateral or transforaminal interbody fusion (LIF or TLIF) with percutaneous pedicle screw fixation), hybrid techniques (HYB) (MIS LIF or TLIF in combination with open posterior pedicle screw fixation), and open techniques to assess ODI at 1 and 2 years.

Methods: All cases were reviewed retrospectively. Inclusion criteria included: age $>18 \mathrm{yr}, \mathrm{ASD}$, min $2 \mathrm{yr}$ follow-up. Patients treated for adult spinal deformity using either less invasive or open surgical approaches were propensity matched by preop SVA, baseline ODI, and by number of fused levels. Patients' results were compared at 1 and 2 years postop.

Results: 40 patients were propensity matched into each group for a total number of 120 . Mean number of levels fused and SVA was $\mathrm{HYB}=5.9,37.7 \mathrm{~mm}, \mathrm{MIS}=3.7,30.7 \mathrm{~mm}, \mathrm{OPEN}=6.0,47.5$ $\mathrm{mm}$. At baseline, ODI was: $\mathrm{HYB}=69.6, \mathrm{MIS}=49.7$, Open=49.6. At postop 1 year patients reported significantly improved ODI $(\mathrm{P}<0.01$ when compared to baseline) $(\mathrm{HYB}=37.3, \mathrm{MIS}=26.8$, Open=35.6) (between groups $\mathrm{P}>0.05)$. At 2 years the patients maintained improvement in disability (HYB=37.5, SVA 43.6, MIS 28.0, SVA 34.5, and Open 30.6, SVA 33.5).

Conclusions: Patients treated with MIS techniques can achieve similar reductions in disability to those treated with open deformity surgery. When matched by SVA, number of levels and baseline ODI, there was no statistically significant difference in disability at 1 and 2 years after surgery.

\section{Simulated Long-Term Biomechanical Evaluation of Revision Surgical Techniques in the Event of Long Fusion Implant Failure}

Seth Shawn Molloy DO MSc (University of Miami - Miller School of Medicine), Karthik H. Madhavan MD (University of Miami Hospital), Jonathan Harris MS, Allan D. Levi MD, PhD (University of Miami-Miller School of Medicine), Mir Hussain BS, Sean BS Jenkins (Globus Medical, Inc.), Yiwei Cai, John Hao, Brandon Bucklen PhD (Globus Medical Inc.)

Introduction: Long lumbar fixation is commonly used to address degenerative conditions despite high incidence of screw haloing in weak or osteoporotic bone. Implant failure necessitates revision surgery extending to the sacrum; however optimum intervention is unknown. This preliminary study investigated long-term revision stabilization following simulated in vivo fatiguing within a human cadaveric lumbar model.

Methods: Six specimens (L1-S1) were tested in flexionextension (FE), lateral bending (LB), and axial rotation (AR) on a six degree-of-freedom spine motion simulator. Specimens were initially instrumented with $\varnothing 5.5 \mathrm{~mm}$ bilateral pedicle screws (L2L5). Injured state simulated screw haloing at L5 using an $\varnothing 6.5 \mathrm{~mm}$ tap and $\varnothing 5.5 \mathrm{~mm}$ screw. Spines were divided randomly into three groups $(n=2)$ with revision techniques including $\varnothing 6.5 \mathrm{~mm}$ screws at L5 and (a) integrated anterior lumbar interbody fusion (ALIF) (INDEPENDENCE®, Globus Medical), (b) bicortical $\varnothing 6.5 \mathrm{~mm}$ pedicle screws at S1 (BS1) (REVERE®, Globus Medical), or (c) $\mathrm{BS} 1+\mathrm{ALIF}$. In vivo fatiguing required 18,000 cycles of maximum FE on a MTS servohydraulic machine, simulating six months of implant wear. Constructs tested include: (1) Intact; (2) L2-L5; (3) injured; (4) initial revision; (5) post-fatigue. Motion reported was normalized to intact per specimen from L4-S1.

Results: Average DEXA score for specimens with ALIF, BS1, and BS1+ALIF groups were $-1.65,-0.7$, and -3.1 , respectively. ROM data is found in Figures 1-3. Initial revision successfully reduced motion relative to the injured state, while fatigue increased motion across all constructs, in all motion planes. BS1 provided the most fixation relative to injured and was the most stable construct prior and following fatigue.

Conclusions: Preliminary results show BS1 provided superior fixation both prior and following fatigue. ALIF provided the least amount of fixation while rigidity of BS1+ALIF may cause further damage in patients with weak or osteoporotic bone. Future clinical studies are required to determine if these differences impact patient outcome. 
250. Does the Use of Minimally Invasive Pedicle Screw Fixation Lower the Rate of PJK and Reoperation for Proximal Junctional Failure (PJF)

Paul Park MD (University Of Michigan, Department of Neurosurgery), Praveen V. Mummaneni MD (University of California San Francisco Spine Center), Gregory Mundis MD (San Diego Center for Spinal Disorders), Neel Anand MD, Juan S. Uribe MD (University of South Florida - Division of Neurosurgery), Michael Y. Wang MD FACS (University of Miami), Adam S. Kanter MD (University of Pittsburgh Medical Center Presbyterian), Richard G. Fessler MD, Stacie Nguyen MPH, Christopher I. Shaffrey MD FACS (University of Virginia), Kai-Ming G. Fu MD PhD (Weill Cornell Medical College)

Introduction: Use of minimally invasive (MIS) pedicle screw fixation has been theorized to lower the PJK rate by reducing paraspinal soft tissue injury. The purpose of this study was to compare rates of PJK and reoperations for PJF in propensity matched cohorts who had MIS versus open pedicle screw placement to correct moderate adult deformity.

Methods: Two multi-center databases were queried. Inclusion criteria were age $>18$ yrs and one of the following criteria: coronal scoliosis $=20^{\circ}$, SVA $>5 \mathrm{~cm}, \mathrm{PT}>25^{\circ}$, or thoracic kyphosis $>60^{\circ}$. Patients were categorized into 3 groups by surgical approach. The MIS group were those who had lateral interbody fusion (LIF) and/ or MIS TLIF with posterior percutaneous instrumentation. Hybrid (HYB) group were those who had LIF followed by open posterior instrumentation. Open (OPEN) group were those who had a traditional posterior exposure for screw placement $+/$ - osteotomies. Patients were propensity matched for SVA, PI-LL mismatch, and levels fused. 114 patients were included with 38 in each group. All patients had 2 year minimum follow-up. PJK was defined as proximal junctional angle $>10^{\circ}$ and change post-op $>10^{\circ}$.

Results: Mean age was 60.8(MIS), 62.4(HYB), and 53.5(OPEN) yrs $(\mathrm{p}=0.018)$. Pre-op SVA and PI-LL were similar and remained so at 2 year follow-up (Table 1). Mean levels fused were 4.7(MIS), 5.4(HYB), 6.8(OPEN) $(\mathrm{p}=0.002)$. Radiographic PJK rates were similar. However, $0(0 \%)$ cases in MIS vs $6(15.8 \%)$ in HYB $(\mathrm{p}=0.01)$ and vs $3(7.9 \%)$ in OPEN $(\mathrm{p}=0.07)$ required re-operation. Mean PJK angle in these patients were $21.3^{\circ}$ for HYB and $23.1^{\circ}$ for OPEN.

Conclusions: In this comparative study, MIS pedicle screw fixation resulted in a similar rate of radiographic PJK in patients who were propensity matched for SVA and PI-LL mismatch (pre-op SVA $<5 \mathrm{~cm}$, PI-LL of 10-20 degrees) and levels fused. There was a trend toward MIS cases requiring less reoperation for PJK.

251. The Clinical Correlation of the Hart-ISSG Proximal Junctional Kyphosis Severity Scale (PJKSS) with Health Related Quality of Life Outcomes and Need for Revision Surgery

Darryl Lau MD (University of California, San Francisco), Haruki Funao MD (Johns Hopkins University), Aaron J. Clark MD PhD (Semmes-Murphey Neurologic \& Spine Institute), Fred Nicholls, Justin S. Smith MD PhD (University of Virginia Health System), Shay Bess MD (Rocky Mountain Scoliosis and Spine Center), Christopher I. Shaffrey MD FACS (University of Virginia), Frank Schwab MD, PhD, Virginie Lafage PhD (NYU Langone Medical Center), Vedat Deviren MD (Univeristy of California San Francisco), Robert Hart MD (Ohsu Spine Center), Khaled Kebaish $M D$, Christopher P. Ames MD (University of California San Francisco Neuro Surgery)

Introduction: Proximal junctional kyphosis (PJK) and proximal junctional failure (PJF) are well-described adverse outcomes following long-segment instrumented fusion for spinal deformities. Hart and the International Spine Study Group (ISSG) developed a PJK severity scale (Hart-ISSG PJKSS) which incorporates neurological deficit, pain, instrumentation problems, degree of kyphosis, presence of fracture, and level of upper-most instrumented vertebrae. This study evaluated the correlation between Hart-ISSG PJKSS and quality of life measures and the need for revision surgery in patients with PJK.

Methods: All adult spinal deformity patients with PJK and/or PJF were identified from two large academic centers over a 7-year period. Health related quality of life (HRQOL) measures were prospectively collected at the time of PJK diagnosis: Oswestry Disability Index (ODI), visual analogue scale (VAS) pain, SF-36 questionnaire, and SRS-30 questionnaire. Correlation between the Hart-ISSG PJKSS and HRQOL outcomes and need for revision surgery were assessed.

Results: A total of 184 cases were included. $21.2 \%$ were male and mean age was 65.0 years. $11.4 \%$ presented with weakness and/ or myelopathy. $88.6 \%$ had pain, (mean VAS of 5.1 ). $44.0 \%$ had instrumentation issues and $64.1 \%$ had fractures. PJK occurred in the upper thoracic spine in $21.7 \%$ of cases. Mean Hart-ISSG PJKSS score was 5.9 (range 1 to 15). The Hart-ISSG PJKSS was significantly and strongly associated with ODI $(\mathrm{p}<0.001, \mathrm{r}=0.611)$, VAS pain $(\mathrm{p}<0.001, \mathrm{r}=0.676)$, SRS-30 function $(\mathrm{p}<0.001, \mathrm{r}=-0.401)$, SRS-30 mental health $(\mathrm{p}<0.001, \mathrm{r}=-0.592)$, SRS-30 self-image $(\mathrm{p}<0.001, \mathrm{r}=-0.511)$, SRS-30 satisfaction $(\mathrm{p}<0.001, \mathrm{r}=-0.531)$, and SRS-30 pain $(\mathrm{p}<0.001, \mathrm{r}=-0.445)$. Higher Hart-ISSG PJKSS scores were associated with higher proportion of patients undergoing revision surgery $(\mathrm{p}<0.001)$.

Conclusions: The Hart-ISSG PJKSS was strongly correlated with validated functional outcomes. Higher scores were associated with higher rates of revision surgery. The Hart-ISSG PJKSS may be a useful clinical tool to identify those patients who will ultimately require revision surgery.

252. Recovery Kinetics in 149 Adult Spinal Deformity Patients with 2-year Follow-up: The Effect of Complications and Reoperation Using an Area Under the Curve Analysis

Justin K Scheer BS (University of California, San Diego), Gregory Mundis MD (San Diego Center for Spinal Disorders), Eric Klineberg MD (University of California - Davis), Robert Hart MD (Ohsu Spine Center), Michael P Kelly MD (Washington University), Vedat Deviren MD (Univeristy of California San Francisco), Douglas $C$. Burton MD, Ian McCarthy PhD (4708 Alliance Blvd.), Shay Bess MD (Rocky Mountain Scoliosis and Spine Center), Frank Schwab $M D, P h D$, Christopher I. Shaffrey MD FACS (University of Virginia), Virginie Lafage PhD (NYU Langone Medical Center), Justin S. Smith MD PhD (University of Virginia Health System), Christopher P. Ames MD (University of California San Francisco Neuro Surgery)

Introduction: Complication rates in adult spinal deformity (ASD) surgery are high and the effects on overall recovery are not well understood. Current methods of reporting outcomes are limited to static outcome time points perhaps diminishing the health impact of the entire recovery experience. This study aims to identify the effect of complications on the kinetics of the recovery process (recovery kinetics) by examining the effect of HRQOL over time via an area under the curve analysis (AUC).

Methods: A retrospective review of a multicenter, prospective ASD database. Inclusion criteria, $=18 \mathrm{yrs}$, ASD. Complication number, type, and need for reoperation (reop) were recorded. Pts were stratified as having no complication (NOCOMP) and any complication (COMP). HRQOL collected included Oswestry Disability Index (ODI), Short Form-36(SF-36), and Scoliosis Research Society-22 (SRS22) at baseline, 6wks, 1 and 2yrs postop. All HRQOL was normalized to each pts' baseline scores as a comparison relative to where the pts started. An AUC was then calculated across the entire 2yrs. Standard HRQOL, normalized HRQOL, and AUC means were compared between groups. 


\section{Meeting Abstracts: AANS/CNS Section on Disorders of the Spine and Peripheral Nerves}

Results: 149 pts were included (COMP:84, NOCOMP:45). There were no significant HRQOL differences at any time point between the groups ( $p>0.05$ for all). However, after normalizing the HRQOL, COMP had significantly lower SRS mental scores at 1yr $(1.1 \pm 0.2 \mathrm{vs} 1.2 \pm 0.3, \mathrm{p}=0.0002)$ and $2 \mathrm{yrs}(1.1 \pm 0.3 \mathrm{vs} 1.3 \pm 0.4, \mathrm{p}=0.0003)$. COMP had significantly lower SRS Mental AUC than NOCOMP $(3.2 \pm 0.5 \mathrm{vs} 3.5 \pm 0.6, \mathrm{p}=0.0023) .28(19 \%)$ pts had a reop. There were no significant differences between any HRQOL $2 \mathrm{yr}$ AUC values between pts with complications requiring reop and those with no reop ( $p>0.05$ for all).

Conclusions: Static HRQOL analysis would suggest there is no difference between complication groups. Area under the curve (AUC) analysis suggests there is a significantly protracted mental recovery phase associated with patients that have at least one complication and the addition of reop does not affect the recovery kinetics.

253. A Prospective, Multi-Center Matched and Unmatched Cohort Assessment of Outcomes for Operative and Nonoperative Treatment in Adult Spinal Deformity (ASD)

Tamir Ailon, Dominic Maggio, Justin S. Smith MD PhD (University of Virginia Health System), Virginie Lafage PhD (NYU Langone Medical Center), Christopher I. Shaffrey MD FACS (University of Virginia), Frank Schwab MD, PhD, Richard A. Hostin MD (Southwest Scoliosis Institute / Baylor Scoliosis Center), Oheneba Boachie-Adjei MD, Justin K Scheer BS (University of California, San Diego), Behrooz A. Akbarnia MD, Eric Klineberg MD (University of California - Davis), Munish Gupta MD (University of California - Davis; Ortho Surgery), Vedat Deviren $M D$ (Univeristy of California San Francisco), Robert Hart MD (Ohsu Spine Center), Douglas C. Burton MD, Shay Bess MD (Rocky Mountain Scoliosis and Spine Center), Christopher P. Ames MD (University of California San Francisco Neuro Surgery)

Introduction: Adults with spinal deformity present with pain and disability. Our objective was to compare outcomes for op and nonop treatment for ASD based on a prospective, multi-center patient population.

Methods: Multicenter, prospective analysis of consecutive ASD patients. Inclusion criteria: age $>18$ yr and ASD. Propensity scores were used to match op and nonop patients based on baseline (BL) ODI, SRS22, maximum thoracolumbar/lumbar Cobb angle, pelvic incidence to lumbar lordosis mismatch (PI-LL), and leg pain numeric rating scale (NRS) score.

Results: 689 patients met criteria, including 286 op and 403 nonop, with mean ages of 53 and 55yrs, minimum 2-yr follow-up rates of $86 \%$ and $55 \%$, and mean follow-up of 24.7 and 24.8 months, respectively. At BL, compared with nonop, op patients had significantly worse HRQL based on ODI, SRS22, SF36, and leg and back pain NRS $(p<0.001)$ and had worse deformity based on pelvic tilt, PI-LL, and C7SVA ( $<<0.002)$. Before reaching min 2-yr follow-up 38 nonop patients converted to op treatment and were analyzed in the op group. At min 2-yr follow-up all HRQL measures assessed significantly improved for op patients $(\mathrm{p}<0.001)$, but none of these measures improved significantly for nonop patients $(\mathrm{p}>0.11)$. 97 matched op-nonop pairs were identified based on propensity scores. At last follow-up the 97 matched op patients had significant improvement in all HRQL measures assessed ( $\mathrm{p}<0.001)$, but the 97 matched nonop patients lacked significant improvement in any of the HRQL measures ( $p>0.20)$. Paired op-nonop analysis demonstrated the op patients to have significantly better HRQL scores at follow-up for all measures assessed $(\mathrm{p}<0.001)$, except SF36 MCS $(\mathrm{p}=0.058)$. Operative overall minor and major complication rates were $53 \%$ and $40 \%$, respectively.

Conclusions: Op treatment for ASD can provide significant improvement of HRQL measures at min 2-yr follow-up. In contrast, nonop treatment appears to at best maintain presenting levels of pain and disability.
254. Biomechanical Comparison of Cortical Screws to Traditional Pedicle Screws with Immediate and Simulated Long-term Stabilization Results in a Lumbar Laminectomy Model

Kris Radcliff MD, Noelle F Klocke MS (Globus Medical, Inc), Jonathan Harris MS, Yiwei Cai, Omar Elkassabany, John Hao, Brandon Bucklen PhD (Globus Medical Inc.)

Introduction: Cortical screws (CS) are a shorter, less-invasive posterior stabilization option than pedicle screws (PS), but their stabilization equivalency remains uncertain. Immediate and simulated in vivo fatiguing stabilization results were investigated between traditional PS and more midline, muscle-sparing CS.

Methods: DEXA-matched (+/-0.1) pairs of fresh-frozen cadaveric spines ( $n=14, \mathrm{~L} 1-\mathrm{S} 1)$ were randomly assigned to receive either all $5.0 / 6.0 \mathrm{~mm}$ CS $(25-35 \mathrm{~mm})$, or traditional $5.5 \mathrm{~mm}$ PS $(30-55 \mathrm{~mm}$, Globus Medical, Inc.). Following screw placement, surgical constructs tested included: 1) intact, 2) intact with posterior instrumentation (P), 3)L3-L5 laminectomy, 4) laminectomy+P (L+P), 5) laminectomy+discectomy, 6) laminectomy+discectomy+P, 7) laminectomy+ static TLIF PEEK interbody spacer $+\mathrm{P}(\mathrm{L}+\mathrm{S}+\mathrm{P}$, Globus Medical, Inc.). Range of motion (ROM) data was collected using Optotrak Certus software and a six degree-of-freedom spine motion simulator $\left(7.0 \mathrm{~N}-\mathrm{m}, 1.5^{\circ} / \mathrm{sec}\right)$. Several weeks of in vivo wear $(18,000$ cycles, $0.6-0.75 \mathrm{~Hz})$ was simulated on a mechanical testing system using displacement-controlled loading from $\mathrm{L}+\mathrm{S}+\mathrm{P}$ 's maximum flexion. ROM was collected after fatiguing, then screw pull-out was performed along the screw's major axis $(5 \mathrm{~mm} / \mathrm{min})$. Statistics revealed significant differences in ROM and screw pullout strength $(\mathrm{p}<0.05)$.

Results: A multifactorial ANOVA with repeated measures analysis (differences between screw types) showed that CS permitted significantly more motion in lateral bending across all constructs with $\mathrm{P}(\mathrm{p}<0.05)$. A one-way ANOVA with repeated measures (differences between constructs, within a screw type) revealed no significant differences in any mode of motion when interbody spacers were added $(\mathrm{L}+\mathrm{S}+\mathrm{P})$ in comparison to laminectomy alone $(\mathrm{L}+\mathrm{P})$. A student t-test revealed that average pullout load-to-failure for CS $(690.2 \pm 362.0 \mathrm{~N}, \mathrm{n}=34)$ was significantly lower than PS $(944.2 \pm 390.0 \mathrm{~N}, \mathrm{n}=39, \mathrm{p}<0.01)$; both groups had $-1.7 \pm 0.9$ DEXA scores.

Conclusions: Following simulated wear, cortical screws had less resistance to pullout strength versus traditional pedicle screws, but performed equivalently in stabilizing the lumbar spine in flexionextension and axial rotation during normal loading conditions.

255. Assessment of Impact of Long-Cassette Standing X-rays on Surgical Planning for Lumbar Pathology: An International Survey of Spine Surgeons

Justin S. Smith MD PhD (University of Virginia Health System), Christopher I. Shaffrey MD FACS (University of Virginia), Virginie Lafage PhD (NYU Langone Medical Center), Frank Schwab MD, PhD, Regis W. Haid MD (Atlanta Brain and Spine Care), Themistocles Protopsaltis MD, Eric Klineberg MD (University of California - Davis), Justin K Scheer BS (University of California, San Diego), Vedat Deviren MD (Univeristy of California San Francisco), Robert Hart MD (Ohsu Spine Center), Shay Bess MD (Rocky Mountain Scoliosis and Spine Center), Paul M. Arnold MD (Department of Neurosurgery), Jens Chapman MD (University of Washington), Michael G. Fehlings MD PhD FRCSC FACS (Toronto Western Hospital), Christopher P. Ames MD (University of California San Francisco Neuro Surgery)

Introduction: Surgical planning to address significant lumbar spine pathology, performed without appreciation of global spinal alignment, may have negative consequences. Our objective was 


\section{Meeting Abstracts: AANS/CNS Section on Disorders of the Spine and Peripheral Nerves}

to assess whether the extent of recommended surgery for lumbar pathology would significantly change with the addition of longcassette standing x-rays.

Methods: This was an international on-line survey of spine surgeons. A series of 15 cases of lumbar spine pathology were presented with a brief clinical vignette and lumbar imaging (x-rays and MRI/CT). Surgeons were asked to select the most appropriate surgical plan, with 5 choices, ranging from least aggressive (decompression alone; 1 point) to the most aggressive (upper throracic to sacrum/ilium fusion +/-osteotomies/ decompression/ interbodies; 5 points). Cases were then re-ordered and presented with long-cassette standing $\mathrm{x}$-rays and the same surgical planning question. Results were compared based on lumbar imaging only vs addition of longcassette x-rays. 5 cases (control group) had normal global alignment and 10 cases (study group) had global malalignment.

Results: 316 surgeons completed the survey, predominantly (63\%) from North America and Europe. Specialties included orthopedic surgery $(65 \%)$ and neurosurgery $(34 \%), 68 \%$ completed spine fellowship, and responders had a mean $13.4 \mathrm{yrs}$ in practice that was a mean of $76 \%$ spine and included a mean of 123 fusions per yr. For study cases, extent of recommended surgery increased significantly with addition of long-cassette $\mathrm{x}$-rays vs lumbar imaging only ( $p=0.002$ ). For control cases with normal global alignment, no significant changes in surgery plans were identified with addition of long-cassette $\mathrm{x}$-rays $(\mathrm{p}=0.280)$.

Conclusions: Long-cassette standing $\mathrm{x}$-rays can have significant impact on surgical planning for lumbar pathology. Surgeons should maintain a relatively low threshold for obtaining long-cassette standing $\mathrm{x}$-rays when contemplating surgical treatment for significant lumbar spine pathology.

256. Radiographic Fate of S2-Alar-Iliac Screws after 12-Month Minimum Follow-Up

Marcus D. Mazur MD (University of Utah), Mark Alexander Mahan MD (University of Utah), Lubdha Shah, Andrew T. Dailey

Introduction: Lumbopelvic fixation with S2-alar-iliac (S2AI) screws is a recently developed technique used to improve fusion rates. The S2AI screw crosses the cortical surfaces of the sacroiliac joint with the tip positioned in the dense bone overlying the sciatic notch. This trajectory increases screw purchase, which may improve its biomechanical strength. We report radiographic outcomes in patients who underwent lumbopelvic fixation with S2AI screws with a minimum 12-month follow-up.

Methods: A retrospective review was conducted at the University of Utah of adult patients who underwent lumbopelvic fixation with S2AI screws. Patients underwent CT scans both preoperatively and postoperatively at 12 months or longer. CT scans were reviewed to determine: S2AI screw backout or breakage, peri-screw lucency, sacroiliac joint fusion status and L5-S1 fusion status. Radiographic fusion was defined as the presence of solid bony bridging across cortical surfaces.

Results: Postoperative CT evaluation of 26 S2AI screws was performed on 13 patients [mean follow-up 24.8 months (14 to 52 months)]. Nine patients had L5-S1 interbody grafts. Evidence of loosening was identified in 5 S2AI screws (19\%) in 3 patients $(23 \%)$. Screw lucency was partial, occurring only at the tip in the iliac region in 3 screws and in the sacral region in 2 screws. No lucency involved the full screw length (both sacral and iliac regions). L5-S1 nonunion was seen in 1 patient (8\%), who had evidence of bilateral screw loosening of the sacral regions. Two patients with screw loosening had L5-S1 fusion. No patients had fusion across the sacroiliac joint, developed screw backout or breakage, or required reoperation for L5-S1 nonunion or S2AI-screw related complication.

Conclusions: Patients with S2AI screws demonstrated a high rate of L5-S1 fusion (92\%), no evidence of sacroiliac joint fusion, no major screw-related complications, and low rates of partial periscrew lucency.

257. Sacro-Iliac Joint Fusion with Two Different Minimally Invasive Techniques: Posterior vs. Trans-Articular. Biomechanical Analysis

Hector Enrique Soriano-Baron MD (National Institute of Neurology and Neurosurgery), Nestor Gregorio RodriguezMartinez MD (Hospital Hispano Americano), Eduardo Martinez del Campo Oviedo MD (Barrow Neurological Institute), Phillip M. Reyes BSE, Anna Newcomb (Barrow Neurological Institute), Scott Yerby, Derek Lindsey, Nicholas Theodore MD (Barrow Neurosurgical Associates, Ltd), Neil R. Crawford PhD

Introduction: The sacroiliac joint can be fused using a minimally invasive procedure with either posterior or trans-articular placement of the fusion devices. The biomechanics comparing these placements have not been reported. In this in vitro study, the ranges of motion (ROM) of normal, destabilized and fused SI joints were investigated.

Methods: Seven fresh frozen cadaveric specimens $(5 \mathrm{M} / 2 \mathrm{~F}$, mean age $51.9 \pm 14.4$ years, mean BMD at L4 0.905 $\pm 0.345 \mathrm{~g} / \mathrm{cm} 2$ ) including L4-sacrum-pelvis were included. The ROM during single leg stance and applications of pure moments $(7.5 \mathrm{Nm})$ simulating flexion-extension (FE), lateral bending (LB) and axial rotation (AR) were determined for intact, destabilized specimens (cut Pubic Symphysis [PS]), treated (three $7.0 \mathrm{~mm}$ iFuse Implants; SI-BONE, San Jose, CA) and after the treatment devices were removed. Posterior and trans-articular placements were performed on right and left sides in a randomized order. Analyses were done using paired Student's t-tests.

Results: Cutting the PS significantly increased the SI joint ROM compared to normal in all directions of loading $(\mathrm{p}<.003)$. Based on normalized ROM (Fig. 1), fusion treatment significantly decreased the mobility compared to destabilized in all directions of loading using posterior placement $(\mathrm{p}<.04)$, and during F-E and AR using trans-articular placement $(\mathrm{p}<.02)$. The reduction was nearly significant during LB using trans-articular placement $(\mathrm{p}=.055)$. There were no significant differences in ROM between posterior and trans-articular placements, for any condition ( $p>$.2). Removal of the implants restored the mobility to levels before treatment, for both approaches ( $\mathrm{p}>.08)$.

Conclusions: Treatment of a simulated instability of the sacroiliac joint with the use of three fusion devices significantly decreased the mean range of motion in all directions of loading. There does not seem to be a biomechanical advantage with a posterior or transarticular placement.

Disclaimer: The Journal of Neurosurgery Publishing Group (JNSPG) acknowledges that the preceding abstracts and poster sessions are published verbatim as submitted and did not go through either the JNSPG's peer-review or editing process. 